\title{
Shape preserving design of thermo-elastic structures considering geometrical nonlinearity
}

Zhu, Jihong; Li, Yu; Wang, Fengwen; Zhang, Weihong

Published in:

Structural and Multidisciplinary Optimization

Link to article, DOI:

$10.1007 / \mathrm{s} 00158-020-02532-4$

Publication date:

2020

Document Version

Peer reviewed version

Link back to DTU Orbit

Citation (APA):

Zhu, J., Li, Y., Wang, F., \& Zhang, W. (2020). Shape preserving design of thermo-elastic structures considering geometrical nonlinearity. Structural and Multidisciplinary Optimization, 61, 1787-1804.

https://doi.org/10.1007/s00158-020-02532-4

\section{General rights}

Copyright and moral rights for the publications made accessible in the public portal are retained by the authors and/or other copyright owners and it is a condition of accessing publications that users recognise and abide by the legal requirements associated with these rights.

- Users may download and print one copy of any publication from the public portal for the purpose of private study or research.

- You may not further distribute the material or use it for any profit-making activity or commercial gain

- You may freely distribute the URL identifying the publication in the public portal

If you believe that this document breaches copyright please contact us providing details, and we will remove access to the work immediately and investigate your claim. 


\title{
Shape preserving design of thermo-elastic structures considering geometrical nonlinearity
}

\author{
Jihong Zhu ${ }^{\mathrm{a}, \mathrm{b}, *}, \mathrm{Yu} \mathrm{Li}^{\mathrm{a}}$, Fengwen Wang ${ }^{\mathrm{c}}$, Weihong Zhang ${ }^{\mathrm{a}}$ \\ ${ }^{a}$ State IJR Center of Aerospace Design and Additive Manufacturing, School of Mechanical Engineering, \\ Northwestern Polytechnical University, Xian, Shaanxi 710072, China \\ ${ }^{b}$ MIIT Lab of Metal Additive Manufacturing and Innovative Design, NPU-QMUL Joint Research Institute, \\ Northwestern Polytechnical University, Xian, Shaanxi 710072, China \\ ${ }^{c}$ Section for Solid Mechanics, Department of Mechanical Engineering \\ Technical University of Denmark, DK-2800 Lyngby, Denmark
}

\begin{abstract}
Thermal stress is an important design factor that will influence structural responses and cause local warping deformations. In this paper, such temperature variation effect is considered in the structural optimization and the shape preserving design approach is extended into thermo-elastic problems to prevent local thermal damages. Based on the weak-coupled thermo-elastic system, nonlinear structural responses at large thermo-mechanical loads are accurately analyzed. The complementary elastic work is minimized to obtain a reasonable rigid structure. Corresponding integrated deformation energy is utilized to calculate the warping deformation accumulated in the incremental thermo-mechanical loading process. Shape preserving effect is then achieved by an additional constraint on the local deformation energy. Through the adjoint method, sensitivity analysis is derived in the coupled field with design-dependent heat conduction and geometrical nonlinearity. In the numerical implementation, an energy interpolation scheme is applied to circumvent numerical instability in low stiffness regions and further modified for multi-material design. Optimization results show that local distortions in thermo-elastic structures are effectively eliminated by the proposed shape preserving design approach.
\end{abstract}

Keywords: Shape preserving design, Geometrically non-linear topology optimization, Thermo-elastic problem, Integrated deformation energy

\section{Introduction}

Structural design using topology optimization is experiencing great popularity in recent years [13]. Especially in the aerospace industry, the topology optimization method shows its innovative and valuable applicability for weight savings and performance improvements [4-6]. In the structural design of hypersonic flight, the aircraft is subjected to aerodynamical heating caused by the

\footnotetext{
${ }^{*}$ Corresponding author

Email address: jh.zhu@nwpu.edu.cn (Jihong Zhu)
} 
boundary layer friction. This process generates heat and consequently, all external surfaces on the aircraft are heated. This, in turn, leads to non-uniform temperature distributions that produce thermal stresses and deformations. Likewise, spacecraft structures are subjected to aerodynamical heating during launch and reentry phases, in addition to intensive solar radiation while operating in space. Thus, in such practical engineering design of thermo-elastic structures, thermal issues are significant and local functional components must be prevented from thermal damages, as illustrated in Figure 1.

The thermal effect on the topology design was introduced and investigated by Rodrigues and Fernandes [7] for the first time. Since then, lots of relevant researches and developments have been made. Existing literature considering thermal influences in topology optimization can be divided into several categories as follows.

- One is to take the thermal force into account. A linear thermal force is defined with a temperature change. Combined with the mechanical force, the mean compliance is assigned as the objective function to take care of both the static or dynamic stiffness and thermal loading effect. This can be seen as a stiffness design, such as Refs. [8-10] in linear statics and Refs. $[11,12]$ in dynamics.

- Another one is to focus on the thermal stress effect. Thermally induced stress is limited and stress-constrained large-scale problems are resolved with computationally efficient design methods. In these frameworks, the min-max stress is set as the objective function or the max stress is constrained with a minimum volume. This can be seen as a strength design, such as Refs [13-15]. In the work of Deng and Suresh [16] and Wu et al. [17], the thermal stress influence on the structural buckling performance is further studied.

- The last one is a compliant mechanism design, such as Refs [18-21]. As broadly applied in Micro-Electro Mechanical Systems (MEMS), advanced structures and actuators involving multiple physics and multiple materials are performed using topology optimization.

From an overview of the research work mentioned above, two key points should be noted.

- Firstly, in most cases, geometrically nonlinear effects are neglected for simplification in analysis and design optimization. However, in practical designs with increasing requirements, the nonlinearity cannot be neglected. Deaton and Grandhi [22] investigates the thermoelastic response of characteristic aerospace structures by comparison of linear and nonlinear analysis results. Results indicate that geometrical nonlinearity manifests as stress stiffening behavior and deformation-dependent load contributions. Hence, nonlinearity plays a significant factor in properly predicting the structural response in an elevated temperature environment. Also in the design of compliant actuator, Sigmund [18] proves that the use of geometrically nonlinear modeling is extremely important when buckling parts appear.

- Secondly, as mostly exists, the temperature field is assumed to be a uniform distribution. The varying temperature distribution or design-dependent thermal condition is seldom considered, except in Refs [18, 19] and [23, 24]. The thermo-elastic design with a constant temperature change is relatively simple in academic research but has many limitations for 
engineering problems. Variant coupling parts between the mechanical load and the thermal load cause difficulties in acquiring converged solutions and fine results. Thus to reduce complexity, one possibility is suggested in [7] to model the problem as a multi-load problem [25] where the thermal load will be just one of the different load cases. As presented in $[10,26]$, purely mechanical and thermal loads are considered as two separate load cases. The objective function is the minimum compliance in the pure mechanical load case and the performance in the thermal environment is constrained.

In this work, a thermo-elastic coupling problem with the geometrical nonlinear analysis is considered to ensure structural functionality. The thermal field is a design-dependent temperature result of steady-state heat conduction. Furthermore, a shape preserving design [26-28] of thermoelastic structures is considered to eliminate local warping deformation caused by the thermal stress and then to maintain the normal operation. Until now, shape preserving design has only been developed in pure mechanical situations. As earlier stated, it is more critical to remove the thermal damage in local regions considering temperature effects. The extension of thermo-elastic structural design is important and nontrivial for practical thermal issues.

The remainder of this paper is organized as follows. Section 2 describes the nonlinear analysis of the thermo-elastic structures considering geometrical nonlinearity. Section 3 presents the definition of the thermo-elastic shape preserving problem. Section 4 introduces the numerical implementations of the design and optimization methods along with the sensitivity analysis. Section 5 presents examples to verify the effectiveness. Conclusions are drawn in Section 6.

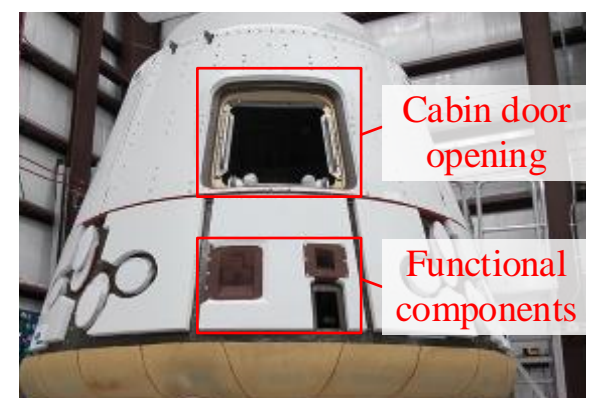

Figure 1: The SpaceX's spacecraft Dragon capsule with a heat shield to withstand extremely high reentry temperatures (picture source from [29])

\section{Formulations of the thermo-elastic problem}

In this section, basic formulations of the finite element model considering nonlinear thermoelasticity are briefly presented. The total Lagrangian approach is employed for the discretized finite element analysis of the static equilibrium. A hyperelastic material model modified with an energy interpolation scheme is further utilized to establish the constitutive relation. For more details, the readers are referred to standard textbooks on finite element methods and continuum mechanics, e.g. Refs. [30-34]. 


\subsection{Non-linear finite element analysis of the thermo-elastic structure}

In the present work, a geometrically non-linear thermo-elastic problem is considered. To this end, the total Lagrangian finite element formulation is applied to derive the kinematics of the thermo-elastic deformation.

Under a thermo-mechanical loading, the initial position of a material particle denoted as $\mathbf{X}$ is assumed to be mapped to the current one denoted as $\mathbf{x}$ after a deformation displacement $\mathbf{u}$ with $\mathbf{x}=\mathbf{X}+\mathbf{u}$. The relation from the undeformed configuration, $\mathcal{S}^{0}$, to the deformed one, $\mathcal{S}$, can be addressed by a total deformation gradient tensor $\mathbf{F}=\partial \mathbf{x} / \partial \mathbf{X}$.

Then, the total Green-Lagrange strain for large displacements is given in tensor form as

$$
\mathbf{E}=\frac{1}{2}\left(\mathbf{F}^{\mathrm{T}} \cdot \mathbf{F}-\mathbf{I}\right)
$$

where $\mathbf{I}$ is the identity tensor and the superscript $\mathrm{T}$ indicates the transpose. For thermally isotropic materials, the elastic or mechanical strain can be derived as $[18,35]$

$$
\mathbf{E}^{\mathrm{m}}=\mathbf{E}-\alpha\left(T-T_{0}\right) \mathbf{I}
$$

where $T$ and $T_{0}$ are the current temperature and reference one for the coefficient of thermal expansion $\alpha(C T E)$, respectively. In this paper, the reference temperature is set as $T_{0}=273.15 \mathrm{~K}=0{ }^{\circ} \mathrm{C}$, and $C T E$ is restricted to a constant in the temperature range of interest (below $50{ }^{\circ} \mathrm{C}$ ) [18]. Therefore, it is assumed that a thermal strain part is a linear form of the temperature while the mechanical one is still nonlinear and dependent on the displacement and temperature.

The separation method of thermal and mechanical strains in Eq. (2) is simple to implement and well adopted in solving thermo-elastic problems undergoing large deformations and small strains $[18,35]$. It is an approximation that is only valid in small thermal strains. For a more general and precise analysis method in nonlinear thermo-elasticity considering both material nonlinearity (large thermal strains) and geometrical nonlinearity (large mechanical displacements), readers are suggested to a multiplicative decomposition method [36-40].

The second Piola-Kirchhoff stress tensor $\mathbf{S}^{\mathrm{m}}$, which is the work conjugate of $\mathbf{E}^{\mathrm{m}}$, is computed as

$$
\mathbf{S}^{\mathrm{m}}=\frac{\partial \phi\left(\mathbf{E}^{\mathrm{m}}\right)}{\partial \mathbf{E}^{\mathrm{m}}}
$$

where $\phi$ denotes a strain energy density function that describes the material behavior. A specific material model will be chosen in the next section.

Using a standard finite element discretization, the discretized nonlinear thermo-elastic system can be governed by two finite element equations such that

$$
\left\{\begin{array}{l}
r^{\text {th }}=\boldsymbol{Q}-\boldsymbol{K}^{\text {th }} \boldsymbol{T}=\mathbf{0}, \\
\boldsymbol{r}^{\mathrm{m}}=\boldsymbol{f}^{\mathrm{ext}}-\boldsymbol{f}^{\mathrm{int}}=\mathbf{0},
\end{array}\right.
$$

where $\boldsymbol{r}^{\text {th }}$ and $\boldsymbol{r}^{\mathrm{m}}$ are the residual vectors of the structural equilibriums for thermal and mechanical analyses, respectively. In Eq. (4a), $\boldsymbol{Q}$ is the external thermal load vector (e.g. heat flux), $\boldsymbol{K}^{\text {th }}$ is the symmetric heat conductivity matrix, which is independent on the nodal temperature and 
displacement. $\boldsymbol{T}$ is the nodal temperature vector. The thermal field is assumed as a linear steadystate heat conduction problem without heat convection or radiation. For nonlinear heat conduction problems, where material properties are temperature-dependent, readers are suggested to Ref. [41]. In Eq. (4b), $f^{\text {ext }}$ is the external mechanical load vector and $f^{\text {int }}$ is the internal nodal force vector given by

$$
\boldsymbol{f}^{\mathrm{int}}=\sum_{e} \frac{\partial \int_{\Omega_{e}} \phi(\boldsymbol{u}) \mathrm{d} \Omega_{e}}{\partial \boldsymbol{u}_{e}},
$$

where $\boldsymbol{u}_{e}$ is the nodal displacement vector of element $e$. All the external forces are assumed to be design-independent. The linear equilibrium, Eq. (4a), can be directly solved and the nonlinear equilibrium, Eq. (4b), is solved using the Newton-Raphson method with the iterative equation given as

$$
\boldsymbol{K}^{\tan } \Delta \boldsymbol{u}=\boldsymbol{r}^{\mathrm{m}},
$$

where $\boldsymbol{K}^{\tan }$ is the tangent stiffness matrix defined as

$$
\boldsymbol{K}^{\tan }=-\frac{\partial \boldsymbol{r}^{\mathrm{m}}}{\partial \boldsymbol{u}} .
$$

In solving the above thermo-mechanical coupling problems, it is assumed that temperatures will influence the mechanical behaviour while conversely, geometrical variations do not influence conduction properties. In such weakly coupled thermal-structural analysis procedure, discrete temperature distribution result is firstly obtained from heat conduction Eq. (4a) and then applied to the structural model to determine its thermo-elastic response. For each temperature distribution, a separate structural analysis of Eq. (4b) is performed based on the temperature. As discussed in [22], this procedure is appropriate for the majority of applications and well adopted in most analyses and designs for coupling problems (see e.g. [18-20, 42-44]).

Note that, during the incremental analysis of Eq. (4b), both of the external thermal and mechanical loads should be simultaneously loaded with the same incremental steps. In the case of pure thermal loading, where a prescribed temperature $T$ is uniformly distributed, only Eq. (4b) is solved to determine the response of the structure.

\subsection{Hyperelastic constitutive model of the thermo-elastic structure}

To exactly describe the material behavior with finite-strain thermo-elasticity, constitutive equations are needed. Formulations and derivations in the previous section assumed an arbitrary elastic material model. In the examples section, the isotropic Saint-Venant-Kirchhoff strain energy density function, as the simplest hyperelastic material formulation, is utilized to model the stress-strain relation. For 2D problems, plane stress assumption is adopted. The stored elastic energy density in the Saint-Venant-Kirchhoff model is solely determined as a function of the elastic strain $\mathbf{E}^{\mathrm{m}}$ [33]. It is given by

$$
\phi_{H}=\frac{1}{2} \lambda\left(\operatorname{tr} \mathbf{E}^{\mathrm{m}}\right)^{2}+\mu \mathbf{E}^{\mathrm{m}}: \mathbf{E}^{\mathrm{m}},
$$

where $\lambda$ and $\mu$ are the Lamé parameters. For simplicity, all the elastic material moduli including Young's modulus $E$ and Poisson's ratio $v$ are assumed to be temperature-independent. Formulas of the relations among these parameters are given as $\lambda=v E /\left(1-v^{2}\right), \mu=E /(2(1+v))$. 
When modeling weak regions generated by a topology optimization process, ill-posedness of the tangent stiffness matrix will arise from the geometrically nonlinear analysis and result in non-convergence of the non-linear finite element solver [45-47]. Such numerical instabilities are induced by excessive deformations in fictitious low stiffness elements. To solve such crucial issues, the effective energy interpolation scheme proposed by Wang et al. [47] is implemented in the hyperelastic model to further perform topology optimization. This energy interpolation form for element $e$ is expressed as

$$
\bar{\phi}\left(\boldsymbol{u}_{e}\right)=\phi_{H}\left(\gamma_{e} \boldsymbol{u}_{e}\right)-\phi_{L}\left(\gamma_{e} \boldsymbol{u}_{e}\right)+\phi_{L}\left(\boldsymbol{u}_{e}\right),
$$

where $\boldsymbol{u}_{e}$ is the elemental nodal displacement vector. $\phi_{H}(\cdot)$ refers to the hyperelastic strain energy density and $\phi_{L}(\cdot)$ is the linear one under small deformation. The interpolation factor $\gamma_{e}$ is defined as a threshold using a smoothed Heaviside projection function stated as

$$
\gamma_{e}=\frac{\tanh \left(\beta_{0} \rho_{0}\right)+\tanh \left(\beta_{0}\left(\bar{\rho}_{e}^{p^{\mathrm{m}}}-\rho_{0}\right)\right)}{\tanh \left(\beta_{0} \rho_{0}\right)+\tanh \left(\beta_{0}\left(1-\rho_{0}\right)\right)},
$$

where $\gamma_{e}$ is 1 for the element with $\bar{\rho}_{e}^{p^{\mathrm{m}}}>\rho_{0}$ and 0 for $\bar{\rho}_{e}^{p^{\mathrm{m}}}<\rho_{0} . \bar{\rho}_{e}^{p^{\mathrm{m}}}$ is the elemental physical density powered by the penalty (to be defined in Sec. 4.1) and indicates the element stiffness. The control parameters in Eq. (10) are fixed to be $\rho_{0}=0.01$ and $\beta_{0}=500$ for all examples as suggested in [47].

With the application of the energy interpolation method, the strain energy density in a solid or intermediate density element corresponds to the nonlinear one, whereas the strain energy density in a weak element simply corresponds to the linear one. As a special case of linearization, the stored elastic strain energy density under small deformation is given as

$$
\phi_{L}=\frac{1}{2} \boldsymbol{\epsilon}^{\mathrm{m}}: \mathbf{C}_{L}: \boldsymbol{\epsilon}^{\mathrm{m}},
$$

where $\mathbf{C}_{L}$ is a rank four tensor whose components are linear elastic moduli. Followed by the Duhamel-Neumann postulate of linear thermo-mechanics [37], the infinitesimal effective strain tensor $\epsilon^{\mathrm{m}}[37]$ is defined as

$$
\boldsymbol{\epsilon}^{\mathrm{m}}=\boldsymbol{\epsilon}-\alpha\left(T-T_{0}\right) \mathbf{I},
$$

where $\boldsymbol{\epsilon}$ is the infinitesimal strain tensor. With the scheme (9) implemented in the strain energy density function of Eq. (5), the nodal internal force vector of element $e$ is further interpolated as

$$
\overline{\boldsymbol{f}_{e}^{\text {int }}}\left(E_{e}, \gamma_{e}\right)=\frac{\partial \int_{\Omega_{e}} \bar{\phi}\left(\boldsymbol{u}_{e}\right) \mathrm{d} \Omega_{e}}{\partial \boldsymbol{u}_{e}}=\gamma_{e} \boldsymbol{f}_{e H}^{\text {int }}-\gamma_{e}^{2} \boldsymbol{f}_{e L}^{\text {int }}+\boldsymbol{f}_{e L}^{\text {int }}=E_{e} \overline{\boldsymbol{f}_{e 0}^{\text {int }}}\left(1, \gamma_{e}\right),
$$

where $f_{e H}^{\text {int }}$ is the nonlinear elemental nodal force as a result of the nonlinear energy density and $f_{e L}^{\text {int }}$ is similarly the linear one. $\overline{f_{e 0}^{\text {int }}}$ is the internal force interpolation result with unit Young's modulus of element $e$. The elemental Young's modulus $E_{e}$ will further be interpolated for topology optimization, which will be discussed in Sec. 4.1. Note that, in the interpolation polynomials of Eq. (9) and Eq. (13), it is assumed that the Young's modulus is separable from the energy function and the strain energy depends linearly on the Young's modulus. 
Potentially, it is straight forward to substitute the simple material model (8) used in this paper with other more advanced hyperelastic material laws (e.g. neo-Hookean [48]), but this is not expected to change the present optimization results in any significant way as discussed in [47]. Despite large deformations, strains in load carrying solid regions stay small. Hence, alternative material models only influence algorithmic convergence in low stiffness elements and not structural performance.

\section{Shape preserving design problem considering geometrically nonlinear thermo-elasticity}

As illustrated in Figure 2, sub-regions are defined as shape preserving domains $\Omega_{s}$, where local warping deformations under mechanical and heat loads are concerned. Based on the previous work [28] in a pure mechanical loading case, the shape preserving design of thermo-elastic structures can be further developed by incorporating the thermo-elastic analysis as stated in Sec. 2.1 into the optimization system. As follows, the design functions and model used in this work are expressed, specific instructions on the formulations considering thermo-elasticity are pointed out.

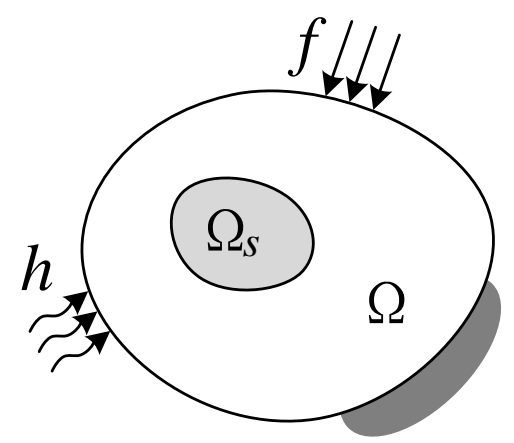

Figure 2: Definition of shape preserving domains subject to heat and mechanical loads

\subsection{Design functions}

In design optimization considering linear thermo-elasticity, the structural mean compliance or elastic strain energy is typically set as the objective function. The differences in the above two functions and design effects are comparatively investigated in Refs. [49-51]. In the linear case, an equivalent thermal force $f^{\text {th }}$ is defined and separable from the internal force $f^{\text {int }}$.

However, as analyzed in Sec. 2, the thermal force is not available in the present nonlinear thermo-elastic problem. Thus, the objective function of the present work is assigned as the complementary elastic work, i.e.

$$
W_{\Omega}^{\mathrm{c}}=\int_{\mathbf{0}}^{\boldsymbol{f}^{\mathrm{ext}}}(\boldsymbol{u}(\boldsymbol{f}))^{\mathrm{T}} \mathrm{d} \boldsymbol{f} \approx \Delta \boldsymbol{f}^{\mathrm{T}}\left(\sum_{i=1}^{n-1} \boldsymbol{u}_{i}+\frac{1}{2} \boldsymbol{u}_{n}\right),
$$

where $n$ is the number of load increments, $\Delta \boldsymbol{f}$ is the increment size determined by $\Delta \boldsymbol{f}=\boldsymbol{f}^{\mathrm{ext}} / n . \boldsymbol{u}_{n}$ is the final displacement vector from the incremental loading process and $\boldsymbol{u}_{i}$ is the corresponding 
displacement vector at the $i$-th load increment. Using this goal, the optimization will result in a stable and stiff structure, where the accumulated structural deformation is minimized within the incremental history up to the given thermo-mechanical loads.

To measure the warpage of the shape preserving domain, the integrated deformation energy function proposed by Li et al. [28] is implemented. The global and local deformation energies are calculated as

$$
\begin{gathered}
\Phi_{\Omega}=\int_{\Omega} \int_{\mathbf{0}}^{\boldsymbol{u}_{n}}\left(\frac{\partial \bar{\phi}(\boldsymbol{u})}{\partial \boldsymbol{u}}\right)^{\mathrm{T}} \mathrm{d} \boldsymbol{u} \mathrm{d} \Omega=\int_{\mathbf{0}}^{\boldsymbol{u}_{n}}\left(\overline{\left.\boldsymbol{f}^{\text {int }}(\boldsymbol{u})\right)^{\mathrm{T}} \mathrm{d} \boldsymbol{u},}\right. \\
\Phi_{\Omega_{s}}=\int_{\Omega_{s}} \int_{\mathbf{0}}^{\boldsymbol{u}_{n}}\left(\frac{\partial \phi_{H}(\boldsymbol{u})}{\partial \boldsymbol{u}}\right)^{\mathrm{T}} \mathrm{d} \boldsymbol{u} \mathrm{d} \Omega_{s}=\int_{\mathbf{0}}^{\boldsymbol{u}_{n}}\left(\boldsymbol{f}_{\Omega_{s} H}^{\mathrm{int}}(\boldsymbol{u})\right)^{\mathrm{T}} \mathrm{d} \boldsymbol{u},
\end{gathered}
$$

where the global deformation energy is calculated with the interpolated strain energy density and the local deformation energy is based on the original hyperelastic one. Note that the integrated deformation energy is defined to calculate the stored energy during the whole loading process, which has no relevance to the strain energy function. A refined criterion for the normalized warpage function is given as

$$
\hat{\Phi}_{\Omega_{s}}=\frac{\Phi_{\Omega_{s}}}{E_{\Omega_{s}}} E_{1},
$$

where $E_{\Omega_{s}}$ is the Young's modulus in the shape preserving domain and $E_{1}$ is the Young's modulus of the base material in the general design domain. This criterion yields a stiffness independent measure of the geometrical distortion, as discussed in [28].

Note that, the deformation energy considering thermo-elasticity is zero when the local domain is freely expanded. Thus, the thermo-elastic shape preserving effect is a local stress-free design, which prevents the local substructure from failure.

\subsection{Design model}

The optimization problem solved in this paper is written as

$$
\begin{array}{cl}
\text { find } & \boldsymbol{\rho}=\left(\rho_{1}, \rho_{2}, \ldots, \rho_{n_{e}}\right)^{\mathrm{T}}, \\
\min & W_{\Omega}^{\mathrm{c}}(\boldsymbol{\rho}), \\
\text { s.t. } & \boldsymbol{r}^{\mathrm{th}}(\boldsymbol{\rho}, \boldsymbol{T})=\mathbf{0}, \\
& \boldsymbol{r}^{\mathrm{m}}(\boldsymbol{\rho}, \boldsymbol{T}, \boldsymbol{u})=\mathbf{0}, \\
& V(\boldsymbol{\rho}) \leq V^{*}, \\
& \hat{\Phi}_{\Omega_{s}}(\boldsymbol{\rho}) / V_{\Omega_{s}} \leq \varepsilon_{\mathrm{sp}}, \\
& \Phi_{\Omega}(\boldsymbol{\rho}) / V^{*} \\
& W_{\Omega}^{\mathrm{cA}}(\boldsymbol{\rho}) \leq W_{\Omega}^{\mathrm{cA}} \\
& 0 \leq \rho_{e} \leq 1 \quad e=1, \ldots, n_{e} .
\end{array}
$$

Here, $\boldsymbol{\rho}$ is the design variable vector containing $n_{e}$ element-based design variables $\rho_{e}$ in the design domain. $W_{\Omega}^{\mathrm{c}}$ is the complementary elastic work of external forces in the total domain $\Omega$. As discussed in Sec. 2.1, in the weakly coupled thermal and mechanical systems of Eqs. (18c) and (18e), 
the thermal problem is assumed independent on the displacement $\boldsymbol{u} . \Phi_{\Omega}$ is the integrated deformation energy over the load history and $\hat{\Phi}_{\Omega_{s}}$ is the normalized one in the shape preserving domain $\Omega_{s}$ [28]. $V$ and $V^{*}$ are the material volumes of the design domain and its prescribed upper bound, respectively. $V_{\Omega_{s}}$ is the volume of the shape preserving domain. $\varepsilon_{\mathrm{sp}}$ is the shape preserving constraint value. $W_{\Omega}^{\text {cA }}$ and $W_{\Omega}^{\text {cA* }}$ are the structural complementary elastic work analyzed in an additional load case and its upper bound, respectively, introduced to avoid isolation of the shape preserving domain in specific cases [28]. For all the complex thermo-elastic problems considered in this paper, the isolation issue is not observed and hence the additional constraint function Eq. (18g) is inactive. For more detailed descriptions and discussions on the shape preserving design model, readers are referred to Refs. [26, 28].

\section{Numerical implementations}

\subsection{Material interpolation scheme}

\subsubsection{Single material}

Using a modified SIMP interpolation scheme [52], the Young's modulus is related to the physical density of element $e, \bar{\rho}_{e}$, in the following expression

$$
E_{e}=E\left(\bar{\rho}_{e}\right)=E_{0}+\bar{\rho}_{e}^{p^{\mathrm{m}}}\left(E_{1}-E_{0}\right),
$$

where $E_{1}$ is the Young's modulus of the solid material in the design domain and $E_{0}$ is the Young's modulus of void, which is non-zero to avoid the singularity of the tangent stiffness matrix (here $\left.E_{\text {void }}=10^{-9} E_{\text {solid }}\right) . p^{\mathrm{m}}$ is the penalty factor used in the mechanical field. The elemental heat conductivity is also interpolated by the above power-law approach as

$$
K_{e}^{\mathrm{th}}=K^{\mathrm{th}}\left(\bar{\rho}_{e}\right)=K_{0}^{\mathrm{th}}+\bar{\rho}_{e}^{p^{\mathrm{th}}}\left(K_{1}^{\mathrm{th}}-K_{0}^{\mathrm{th}}\right),
$$

where $p^{\text {th }}$ is the penalty factor used in the thermal field and similarly the heat conductivity of void is set as $K_{0}^{\text {th }}=10^{-9} K_{1}^{\text {th }}$.

In principle, the power values could take different ones for each physical property. However, for simplicity both penalty factors are set as equal, normally $p^{\mathrm{m}}=p^{\text {th }}=3$ [18].

\subsubsection{Multiple materials}

As an extension, the introduction of multiple materials in the design domain may increase the performance and functionality of the structure, which has been demonstrated for designs of multiphysical actuators [19], thermo-elastic materials [53, 54] and for metamaterial structures [55-57]. Thus, to further promote the structural performance, as well as the shape preserving effect, the material design space can be further extended into multiple material one.

Multiple materials composed of two solids and void are considered here. The three-phase design is achieved by introducing two design variables per element, such as

$$
\text { find } \boldsymbol{\rho}=\left(\boldsymbol{\rho}_{1}, \boldsymbol{\rho}_{2}\right)^{\mathrm{T}} \text {. }
$$


where design variable $\rho_{1}$ determines whether there is material in the element or not and $\rho_{2}$ determines the kind of material in the element. Based on the modified SIMP method, multi-material interpolation scheme can be extended as

$$
\boldsymbol{D}_{e}=\boldsymbol{D}\left(\overline{\boldsymbol{\rho}}_{e}\right)=\boldsymbol{D}_{0}+\bar{\rho}_{e 1}^{p^{\mathrm{m}}}\left[\bar{\rho}_{e 2}^{p^{\mathrm{m}}}\left(\boldsymbol{D}_{1}-\boldsymbol{D}_{0}\right)+\left(1-\bar{\rho}_{e 2}^{p^{\mathrm{m}}}\right)\left(\boldsymbol{D}_{2}-\boldsymbol{D}_{0}\right)\right],
$$

where $\boldsymbol{D}_{e}$ is the elemental interpolated elastic matrix. $\boldsymbol{D}_{0}$ is the elastic matrix of void, $\boldsymbol{D}_{1}$ and $\boldsymbol{D}_{2}$ are the elastic matrixes of solid material 1 and 2, respectively. For any isotropic material $m$, its elastic matrix $\boldsymbol{D}_{m}$ under plane stress assumption is

$$
\boldsymbol{D}_{m}=\frac{E_{m}}{1-v_{m}^{2}}\left[\begin{array}{ccc}
1 & v_{m} & 0 \\
v_{m} & 1 & 0 \\
0 & 0 & \frac{1-v_{m}}{2}
\end{array}\right],
$$

where $E_{m}$ and $v_{m}$ are the Young's modulus and Poisson's ratio, respectively. The design materials in element $e$ decided by the corresponding physical densities are depicted as

$$
\begin{cases}\bar{\rho}_{e 1}=1, \bar{\rho}_{e 2}=1 & \text { Solid material } 1 \text { in element } e \\ \bar{\rho}_{e 1}=1, \bar{\rho}_{e 2}=0 & \text { Solid material } 2 \text { in element } e \\ \bar{\rho}_{e 1}=0 & \text { Void material in element } e\end{cases}
$$

In a similar way of using a simple artificial mixture assumption, the heat conductivity and $C T E$ in element $e$ can be written as

$$
\begin{gathered}
K_{e}^{\mathrm{th}}=K^{\mathrm{th}}\left(\overline{\boldsymbol{\rho}}_{e}\right)=K_{0}^{\mathrm{th}}+\bar{\rho}_{e 1}^{t^{\mathrm{th}}}\left[\bar{\rho}_{e 2}^{p^{\mathrm{th}}}\left(K_{1}^{\mathrm{th}}-K_{0}^{\mathrm{th}}\right)+\left(1-\bar{\rho}_{e 2}^{p^{\mathrm{th}}}\right)\left(K_{2}^{\mathrm{th}}-K_{0}^{\mathrm{th}}\right)\right], \\
\alpha_{e}=\alpha\left(\overline{\boldsymbol{\rho}}_{e}\right)=\bar{\rho}_{e 2} \alpha_{1}+\left(1-\bar{\rho}_{e 2}\right) \alpha_{2} .
\end{gathered}
$$

It should be noted that the interpolation of $C T E$ depends only on $\bar{\rho}_{e 2}$, i.e. the material type [53]. The material physical density $\left(\bar{\rho}_{e 1}\right)$ does not change the $C T E$. Considering the energy interpolation scheme (9) used in the geometrically nonlinear analysis, the interpolation factor $\gamma_{e}$ for multimaterial design can be further modified as

$$
\gamma_{e}=\frac{\tanh \left(\beta_{0} \rho_{0}\right)+\tanh \left(\beta_{0}\left(\bar{\rho}_{e 1}^{p^{\mathrm{m}}}-\rho_{0}\right)\right)}{\tanh \left(\beta_{0} \rho_{0}\right)+\tanh \left(\beta_{0}\left(1-\rho_{0}\right)\right)} .
$$

Thus, the energy interpolation for multiple materials is determined only by the current material stiffness other than the material type.

\subsection{Projection filtering}

To promote discrete final designs, the physical density is represented by a smoothed Heaviside projection function based on the hyperbolic tangent function $[52,58]$, stated as

$$
\bar{\rho}_{e}=\frac{\tanh (\beta \eta)+\tanh \left(\beta\left(\tilde{\rho}_{e}-\eta\right)\right)}{\tanh (\beta \eta)+\tanh (\beta(1-\eta))},
$$


where $\tilde{\rho}_{e}$ is the filtered density of element $e . \beta$ controls the sharpness of the projection and $\eta$ is the threshold value between 0 to 1 . When $\beta \rightarrow \infty$, designs $\left(\bar{\rho}_{e}\right)$ are forced towards pure $0-1$ solution. The filtered density is calculated as

$$
\tilde{\rho}_{e}=\frac{\sum_{k \in N_{e}} w_{e}\left(\boldsymbol{x}_{k}\right) v_{k} \rho_{k}}{\sum_{k \in N_{e}} w_{e}\left(\boldsymbol{x}_{k}\right) v_{k}}
$$

where $\boldsymbol{x}_{k}$ are the center coordinates of element $k . v_{k}$ and $\rho_{k}$ are the corresponding volume and design variable value of element $k$, respectively. $N_{e}$ is the neighborhood of element $e$ within a certain filter radius specified by $N_{e}=\left\{k \mid\left\|\boldsymbol{x}_{k}-\boldsymbol{x}_{e}\right\| \leq r\right\}$, and $w_{e}\left(\boldsymbol{x}_{k}\right)=r-\left\|\boldsymbol{x}_{k}-\boldsymbol{x}_{e}\right\|$.

In all examples in this paper, the radius $r$ in the density filter and the threshold value $\eta$ are set to 3.5 times the average element size and 0.5 , respectively.

\subsection{Continuation strategy}

Since the topology optimization problem is highly non-convex, design results may easily converge to local optima. However, by using a continuation approach, the algorithm could produce consistent designs independent on starting guesses and ensure stable finite element convergences $[18,45]$. A continuation scheme proposed in [47] is applied to both penalization parameters $p^{\mathrm{m}}$ and $p^{\text {th }} \cdot p^{\mathrm{m}}$ and $p^{\text {th }}$ are together raised in steps of 0.05 from 1.0 to 3.0, every 2 iterations when $p^{\mathrm{m}}<2.0$ and every 5 iterations when $p^{\mathrm{m}} \geq 2.0$. To obtain a better $0-1$ convergence, a continuation scheme is also employed to increase $\beta$ in Eq. (28) after $p^{\mathrm{m}}=3.0 . \beta$ is set to 4 at first and updated every 10 iterations to $\beta_{\max }=128$ using $\beta=2 \times \beta$.

During the continuation process of the penalty parameters, material interpolations of Eqs. (19) and (20) in mechanical and thermal fields are not always feasible. Low penalty values (e.g. $p^{\text {th }}<2$ ) could result in non-physical material data. As suggested in [18], the Hashin-Shtrikman bounds should be satisfied for each of the physical properties. However, it is not always easy to find the Hashin-Shtrikman bounds, especially for multi-physical and multi-material design problems. Because the optimization will end with a near 0-1 topology, the final results will satisfy the bounds. Essential for geometrically nonlinear problems, a smoothly converging optimization history is the main focuse in the present research work. It should be mentioned that drawbacks in the absence of physical meanings could change the intermediate optimization path, especially noted for multimaterial interpolations $[19,59,60]$.

\subsection{Sensitivity analysis}

To solve the optimization problems (18), the sensitivities of the structural response to changes in the design variables must be determined. Firstly, the efficient adjoint method is utilized for computing the gradients of all structural functions $\mathcal{F}$ with respect to the elemental physical density $\bar{\rho}_{e}$. $\mathcal{F}$ is a summation function of $\mathcal{F}_{i}$ calculated at every load increment and in each incremental load step, it is augmented with two adjoint vectors, i.e.

$$
\mathcal{F}=\sum_{i=1}^{n}\left[\mathcal{F}_{i}\left(\overline{\boldsymbol{\rho}}, \boldsymbol{T}_{i}, \boldsymbol{u}_{i}\right)+\left(\boldsymbol{\lambda}_{i}^{\mathrm{th}}\right)^{\mathrm{T}} \boldsymbol{r}_{i}^{\mathrm{th}}\left(\overline{\boldsymbol{\rho}}, \boldsymbol{T}_{i}\right)+\left(\lambda_{i}^{\mathrm{m}}\right)^{\mathrm{T}} \boldsymbol{r}_{i}^{\mathrm{m}}\left(\overline{\boldsymbol{\rho}}, \boldsymbol{T}_{i}, \boldsymbol{u}_{i}\right)\right],
$$


where $\lambda_{i}^{\text {th }}$ and $\lambda_{i}^{\mathrm{m}}$ are the adjoint variable vectors in the $i$-th incremental thermal and mechanical load steps, respectively. $\boldsymbol{r}_{i}^{\text {th }}$ and $\boldsymbol{r}_{i}^{\mathrm{m}}$ are the nodal residual force vectors in the thermal and mechanical fields, respectively. $\boldsymbol{T}_{i}$ and $\boldsymbol{u}_{i}$ are the corresponding nodal temperature and displacement vectors at the $i$-th converged solution, respectively.

Thus, all the derivations will also be summation results related to thermal and mechanical load increments, i.e.

$$
\begin{aligned}
\frac{\mathrm{d} \mathcal{F}}{\mathrm{d} \bar{\rho}_{e}}= & \sum_{i=1}^{n}\left[\left(\frac{\partial \mathcal{F}_{i}}{\partial \bar{\rho}_{e}}+\frac{\partial \mathcal{F}_{i}}{\partial \boldsymbol{T}_{i}} \frac{\partial \boldsymbol{T}_{i}}{\partial \bar{\rho}_{e}}+\frac{\partial \mathcal{F}_{i}}{\partial \boldsymbol{u}_{i}} \frac{\partial \boldsymbol{u}_{i}}{\partial \bar{\rho}_{e}}\right)+\right. \\
& \left.\left(\lambda_{i}^{\mathrm{th}}\right)^{\mathrm{T}}\left(\frac{\partial \boldsymbol{r}_{i}^{\mathrm{th}}}{\partial \bar{\rho}_{e}}+\frac{\partial \boldsymbol{r}_{i}^{\mathrm{th}}}{\partial \boldsymbol{T}_{i}} \frac{\partial \boldsymbol{T}_{i}}{\partial \bar{\rho}_{e}}\right)+\left(\lambda_{i}^{\mathrm{m}}\right)^{\mathrm{T}}\left(\frac{\partial \boldsymbol{r}_{i}^{\mathrm{m}}}{\partial \bar{\rho}_{e}}+\frac{\partial \boldsymbol{r}_{i}^{\mathrm{m}}}{\partial \boldsymbol{T}_{i}} \frac{\partial \boldsymbol{T}_{i}}{\partial \bar{\rho}_{e}}+\frac{\partial \boldsymbol{r}_{i}^{\mathrm{m}}}{\partial \boldsymbol{u}_{i}} \frac{\partial \boldsymbol{u}_{i}}{\partial \bar{\rho}_{e}}\right)\right] \\
= & \sum_{i=1}^{n}\left[\frac{\partial \mathcal{F}_{i}}{\partial \bar{\rho}_{e}}+\left(\lambda_{i}^{\mathrm{th}}\right)^{\mathrm{T}} \frac{\partial \boldsymbol{r}_{i}^{\mathrm{th}}}{\partial \bar{\rho}_{e}}+\left(\lambda_{i}^{\mathrm{m}}\right)^{\mathrm{T}} \frac{\partial \boldsymbol{r}_{i}^{\mathrm{m}}}{\partial \bar{\rho}_{e}}+\right. \\
& \left.\left(\frac{\partial \mathcal{F}_{i}}{\partial \boldsymbol{T}_{i}}-\left(\lambda_{i}^{\mathrm{th}}\right)^{\mathrm{T}} \boldsymbol{K}_{i}^{\mathrm{th}}+\left(\lambda_{i}^{\mathrm{m}}\right)^{\mathrm{T}} \frac{\partial \boldsymbol{r}_{i}^{\mathrm{m}}}{\partial \boldsymbol{T}_{i}}\right) \frac{\partial \boldsymbol{T}_{i}}{\partial \bar{\rho}_{e}}+\left(\frac{\partial \mathcal{F}_{i}}{\partial \boldsymbol{u}_{i}}-\left(\lambda_{i}^{\mathrm{m}}\right)^{\mathrm{T}} \boldsymbol{K}_{i}^{\mathrm{tan}}\right) \frac{\partial \boldsymbol{u}_{i}}{\partial \bar{\rho}_{e}}\right],
\end{aligned}
$$

where $\boldsymbol{K}_{i}^{\text {th }}$ is the linear heat conductivity matrix and $\boldsymbol{K}_{i}^{\mathrm{tan}}$ is the symmetric tangent stiffness at the $i$-th converged solution. To eliminate the unknown terms of $\partial \boldsymbol{T}_{i} / \partial \bar{\rho}_{e}$ and $\partial \boldsymbol{u}_{i} / \partial \bar{\rho}_{e}, \boldsymbol{\lambda}_{i}^{\text {th }}$ and $\boldsymbol{\lambda}_{i}^{\mathrm{m}}$ are chosen such that

$$
\boldsymbol{K}_{i}^{\mathrm{th}} \boldsymbol{\lambda}_{i}^{\mathrm{th}}=\left(\frac{\partial \mathcal{F}_{i}\left(\overline{\boldsymbol{\rho}}, \boldsymbol{T}_{i}, \boldsymbol{u}_{i}\right)}{\partial \boldsymbol{T}_{i}}\right)^{\mathrm{T}}+\left(\frac{\partial \boldsymbol{r}_{i}^{\mathrm{m}}\left(\overline{\boldsymbol{\rho}}, \boldsymbol{T}_{i}, \boldsymbol{u}_{i}\right)}{\partial \boldsymbol{T}_{i}}\right)^{\mathrm{T}} \lambda_{i}^{\mathrm{m}}, \quad \boldsymbol{K}_{i}^{\mathrm{tan}} \lambda_{i}^{\mathrm{m}}=\left(\frac{\partial \mathcal{F}_{i}\left(\overline{\boldsymbol{\rho}}, \boldsymbol{T}_{i}, \boldsymbol{u}_{i}\right)}{\partial \boldsymbol{u}_{i}}\right)^{\mathrm{T}}
$$

By solving the above linear systems, the sensitivity of $\mathcal{F}$ with respect to the physical variable $\bar{\rho}_{e}$ is expressed as

$$
\frac{\mathrm{d} \mathcal{F}}{\mathrm{d} \bar{\rho}_{e}}=\sum_{i=1}^{n}\left[\frac{\partial \mathcal{F}_{i}}{\partial \bar{\rho}_{e}}+\left(\lambda_{i}^{\mathrm{th}}\right)^{\mathrm{T}} \frac{\partial \boldsymbol{r}_{i}^{\mathrm{th}}}{\partial \bar{\rho}_{e}}+\left(\lambda_{i}^{\mathrm{m}}\right)^{\mathrm{T}} \frac{\partial \boldsymbol{r}_{i}^{\mathrm{m}}}{\partial \bar{\rho}_{e}}\right]
$$

Considering the energy interpolation scheme used in Eq. (13), $\partial \boldsymbol{r}_{i}^{\mathrm{m}} / \partial \bar{\rho}_{e}$ is further calculated as

$$
\frac{\partial \boldsymbol{r}_{i}^{\mathrm{m}}}{\partial \bar{\rho}_{e}}=-\left(\frac{\partial \boldsymbol{f}_{i}^{\mathrm{int}}}{\partial \bar{\rho}_{e}}+\frac{\partial \boldsymbol{f}_{i}^{\text {int }}}{\partial \gamma_{e}} \frac{\partial \gamma_{e}}{\partial \bar{\rho}_{e}}\right)
$$

where $f_{i}^{\text {int }}$ is the internal nodal force vector in the $i$-th incremental load step.

Finally, the sensitivity of $\mathcal{F}$ with respect to the design variable $\rho_{e}$ is calculated following the chain rule, i.e.

$$
\frac{\mathrm{d} \mathcal{F}}{\mathrm{d} \rho_{e}}=\sum_{k \in N_{e}} \frac{\mathrm{d} \mathcal{F}_{k}}{\mathrm{~d} \bar{\rho}_{k}} \frac{\mathrm{d} \bar{\rho}_{k}}{\mathrm{~d} \tilde{\rho}_{k}} \frac{\mathrm{d} \tilde{\rho}_{k}}{\mathrm{~d} \rho_{e}},
$$

where $\mathcal{F}_{k}$ is the elemental design function of element $k$. Based on the sensitivity analyses, the structure is iteratively updated using the Method of Moving Asymptotes (MMA) [61]. 


\section{Examples}

This section presents two numerical examples to demonstrate the effectiveness of the proposed thermo-elastic shape preserving design method. In the first example, a long beam in a uniform temperature field is considered to theoretically study the thermal influence on the design result with geometrical nonlinearity. In the second example, a more realistic design problem of a sandwich panel in a varying temperature field is solved, where the design-depended temperature distribution adds more complexities to the analysis and optimization process.

Shape preserving design domains are considered as non-designable solid regions. The constraint value of the material volume in the design domain is set as $40 \%$. The optimization procedure is implemented in a MATLAB platform. Numerical solutions are obtained using 10 equally sized load increments. Newton-Rapson iteration is terminated when the maximum relative residual change is less than $10^{-8}$ and the number of Newton-Rapson iteration steps per load increment is mostly below 6 . The optimization loop is terminated when the maximum absolute difference between two consecutive designs (in terms of design variables) is less than 0.01 . The external move limit for updating the design variables in MMA is set as 0.05. CPU time per optimization iteration is around 60 seconds. The starting design guess is a uniform density distribution satisfying the volume constraint in the design domain.

For clear visualizations, only elements with the physical density larger than 0.5 are presented in the design results. Due to the projection approach, very few elements with intermediate densities remain in the optimized results anyway. All the deformed results are shown with a scaling parameter of 1:1.

\subsection{Double clamped beam with single material}

As shown in Figure 3, a long beam with an aspect ratio of 5 is clamped at both ends and subjected to an external force of $f=400 \mathrm{kN}$ downwards at the midpoint of the upper edge. Four rectangular shape preserving domains are located evenly along the centre of the beam. The geometric dimensions of the structure are also shown in Figure 3. The global structure is uniformly heated with a temperature variation $\Delta T$. Accordingly, temperature-induced reaction forces will be created at both ends.

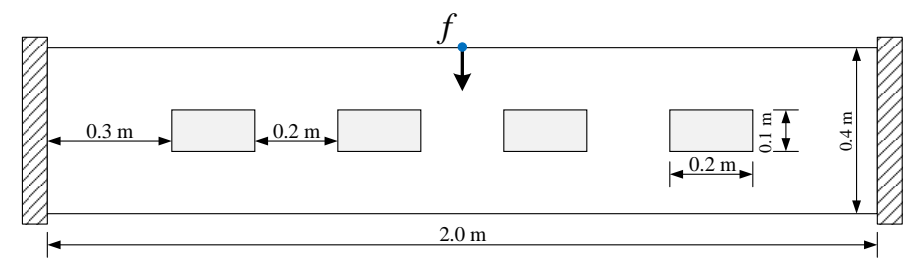

Figure 3: A double clamped beam embedded with four shape preserving domains

The beam structure is discretized using 8000 4-node plane elements with a thickness of 0.1 m. The material properties in the design domain is Young's modulus $E_{1}=3 \mathrm{GPa}$, Poisson's ratio $v_{1}=0.4$ and $C T E \alpha_{1}=20 \times 10^{-6} /{ }^{\circ} \mathrm{C}$. The material property in the four shape preserving domains is $E_{\mathrm{sp}}=0.1 E_{1}=0.3 \mathrm{GPa}, v_{\mathrm{sp}}=v=0.4$ and $\alpha_{\mathrm{sp}}=15 \times 10^{-6} /{ }^{\circ} \mathrm{C}$. 
To provide reference results to be compared with those obtained by considering thermo-elasticity, pure mechanical problems minimizing the global complementary elastic work without and with the shape preserving constraint are solved first without temperature change, i.e. $\Delta T=0{ }^{\circ} \mathrm{C}$. The corresponding (deformed) optimized designs are shown in Figure 4. The optimized objective function and local normalized deformation energy are also stated. In the standard design result without the shape preserving constraint, the objective function $W_{\Omega}^{\mathrm{c}}$ is optimized as $9.134 \mathrm{~kJ}$, while the total normalized warping energy $\hat{\Phi}_{\Omega_{s}}$ in the shape preserving domains is calculated as $7.459 \mathrm{~kJ}$ (corresponding to a relative warping measure of $302 \%$, cf. Eq. (18f)). The deformed configurations (symmetric) of the shape preserving domains are compared to their original shapes indicated by red lines. The normalized deformation energy distribution is also illustrated in the local deformation plots, which further illustrates the effect of the shape preserving measure. It is clearly shown that the sub-domains are undergoing large warping deformations which result in distorted appearances. Under the shape preserving constraint value of $\varepsilon_{\mathrm{sp}}=30 \%$, the shape preserving design is generated to largely eliminate such undesired deformations. The normalized deformation energy in shape preserving domains is then suppressed to $\hat{\Phi}_{\Omega_{s}}=0.795 \mathrm{~kJ}$ with a small increase of the objective function.

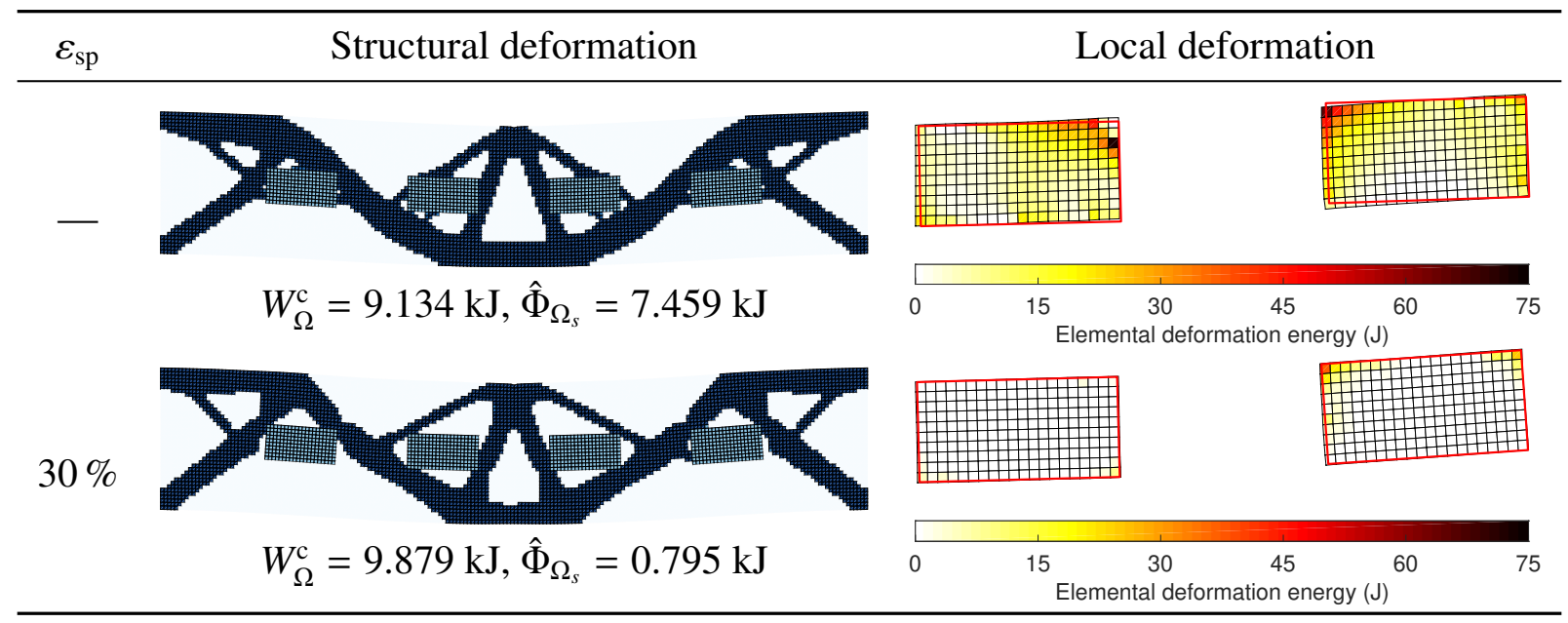

Figure 4: Comparison of design results with single material and a temperature change of $\Delta T=0{ }^{\circ} \mathrm{C}$

Now the structural thermo-elastic performance is examined here by introducing a uniform temperature change. Both standard design and shape preserving design with different temperature changes $\left(\Delta T=50,100^{\circ} \mathrm{C}\right)$ are conducted and corresponding results are compared in Figure 5 and 6, respectively. As illustrated in the shape preserving design results and in contrast to the pure mechanical case, mechanically compressed members (especially the lower bars near the ends) appeared when the temperature change increased. In the elevated temperature condition, contraction stress was added to the compressive parts in the design domain and a stress relaxation was added to the tension parts. Thus, the thermal effect on the structural deformation is manipulated through optimization. Such thermal effect that counteracts the mechanical loading will be further indicated by a multi-material design.

In the standard design results, the local warping deformation was aggravated as well as the 
temperature increased. Local thermal stresses were caused in the connected corners because of different CTEs between the design material and the local one. However, in the shape preserving design result as shown in Figure 6, the local warping deformation is suppressed efficiently by adjusting the configurations according to the benefit of the thermal effect.

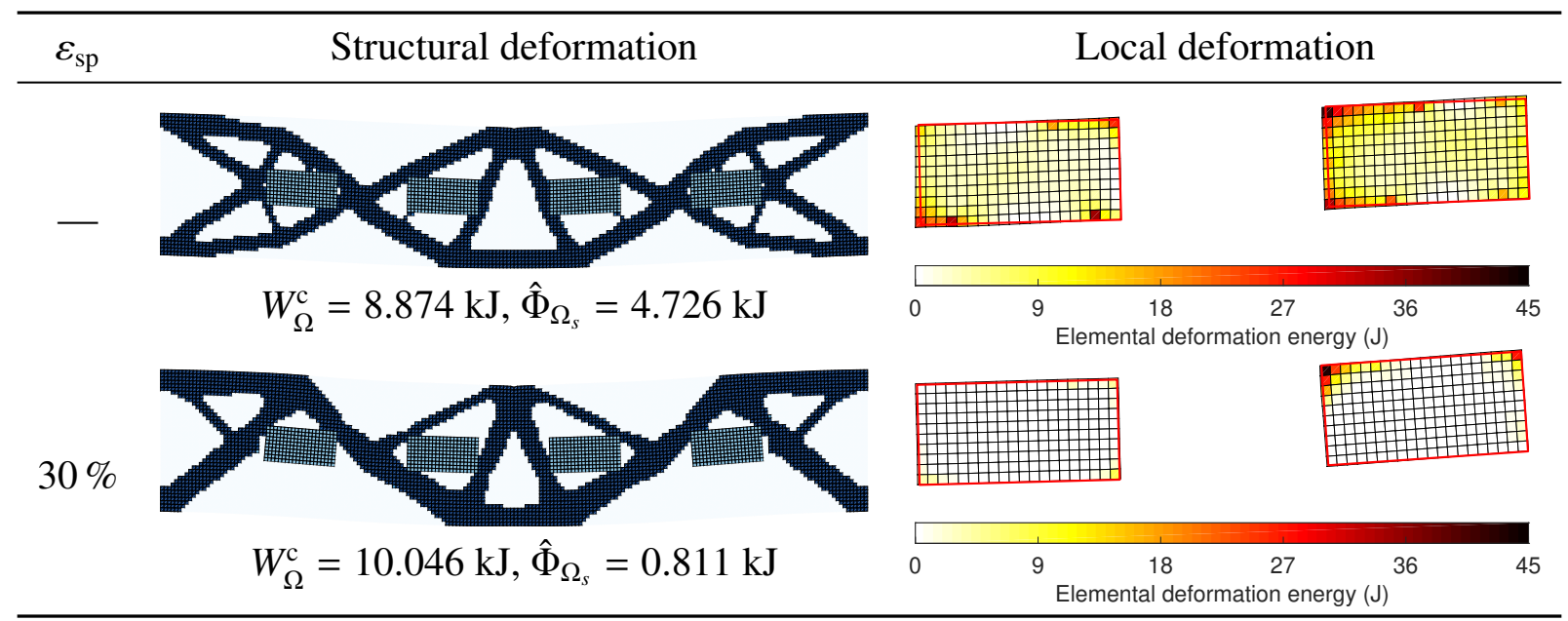

Figure 5: Comparison of thermo-elastic design results with single material and a temperature change of $\Delta T=50{ }^{\circ} \mathrm{C}$

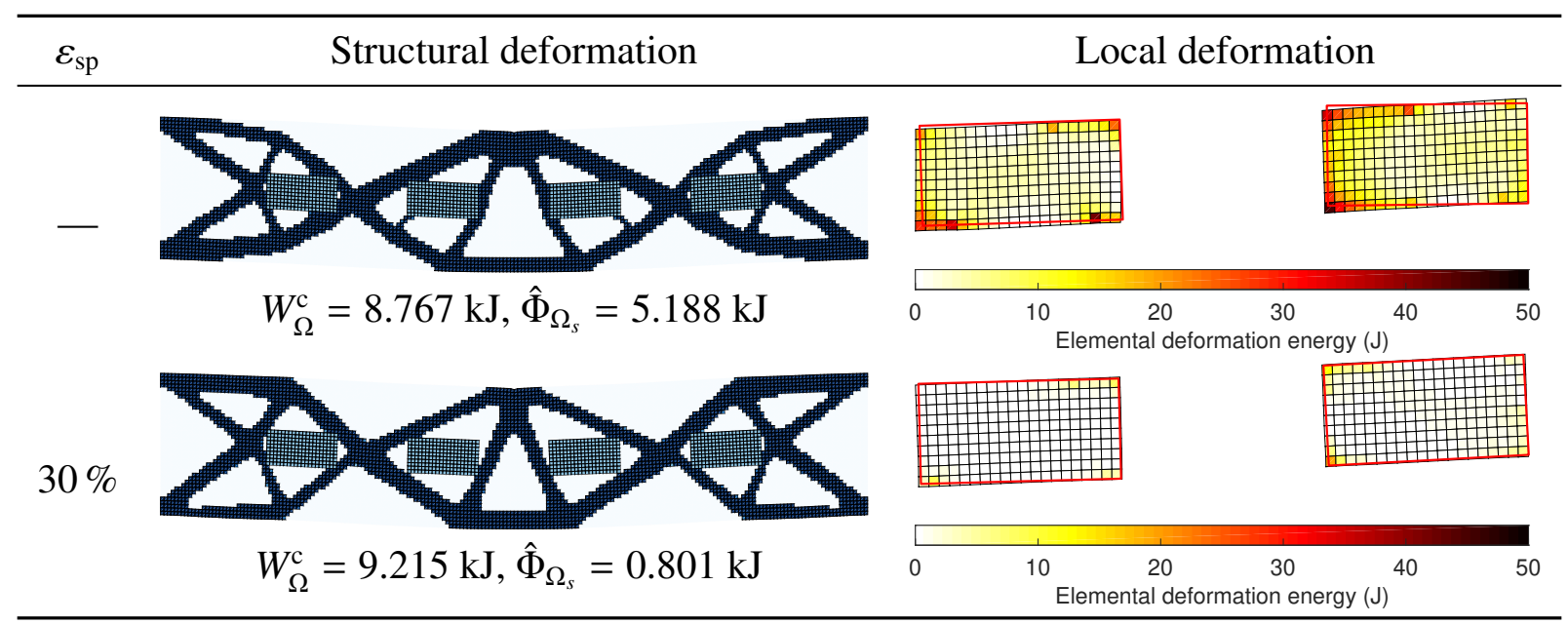

Figure 6: Comparison of thermo-elastic design results with single material and a temperature change of $\Delta T=100{ }^{\circ} \mathrm{C}$

To demonstrate the nonlinear thermal-mechanical coupling effect, standard topology optimizations for the temperature changes of $\Delta T=50,100{ }^{\circ} \mathrm{C}$ are redesigned using linear solutions, respectively. As shown in Figure 7, the linear optimized thermo-elastic layouts are much distinguished from the nonlinear design results. To compare the linear and nonlinear designs, the optimized structures obtained by the linear thermo-elastic topology optimization are analyzed by using the nonlinear thermo-elastic finite element method as also shown in Figure 7. It turns out that the results of the nonlinear responses of the linear layouts are much higher than those of the nonlinear 
layouts, which means the linear design results will generate larger structural deflections and local deformations subject to the same thermo-elastic loads. Although the mechanical nonlinearity is small in the current case, nonlinear thermoelasticity is often significant. Strong deviations of the displacements and deformation energies between the linear and nonlinear designs are shown. Thus, for design accuracy and taking care of possible structural instability such as buckling, nonlinear thermo-elastic consideration is necessary for further shape preserving designs.

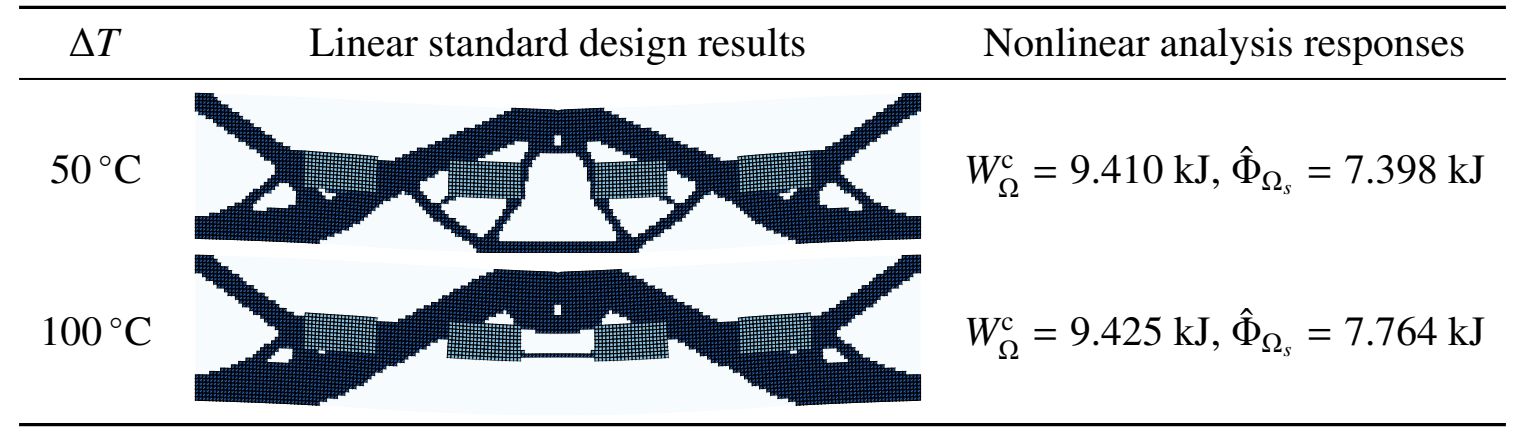

Figure 7: Linear optimized thermo-elastic designs and corresponding nonlinear analysis responses

Next, the decreased temperature change is further studied, which means that the variation of the temperature is negative. As mostly discussed in thermo-elastic researches [7-9, 62], the temperature change is always positive and the mean compliance is set as the objective function to take into account such thermal loading effect. In fact, the temperature change could be negative, as well. For example, an aircraft rises from the ground. Such negative thermal stress has been studied in a design-dependent temperature field in [63], where the thermo-elastic structure is induced by an initial prestress.

The long beam is optimized again with a temperature change of $\Delta T=-100{ }^{\circ} \mathrm{C}$ and the compared design results are presented in Figure 8. In the design results with a negative $\Delta T$, the tension constructed members are highly strengthened so that the cooling effect could shorten the member and hence "pulls up" the structure against the direction of the mechanical load. As similarly observed in [44], the thermally induced deformation always counteracts the mechanically induced displacement in such loading conditions. For the shape preserving design effect, the local deformation is greatly eliminated by modifying the substructures around the sub-domains.

As shown in Figure 9, the optimality performances of the shape preserving design results are checked with different temperature changes. For each of the optimized structure, the objective function and constraint value are reanalyzed, respectively. As expected, taking the case of $\Delta T=$ $-100{ }^{\circ} \mathrm{C}$ for example, it is seen that the optimized design with this temperature change (represented by $\mathbf{\Lambda})$ generates optimal performances among the three structures. Thus, shape preserving design result has been prevented to local optima by the continuation strategy as discussed in Sec. 4.3.

To further demonstrate the shape preserving effect in large deformations, the optimized structures designed with $\Delta T=100,-100^{\circ} \mathrm{C}$ are reanalyzed with a larger external force $(f=2 f=800$ $\mathrm{kN})$. The structural and local deformations of standard design and shape preserving design are compared in Figure 10 and 11, respectively. In the standard design results (a), local deformations are dramatically increased and more geometric distortions are generated in the local regions. 


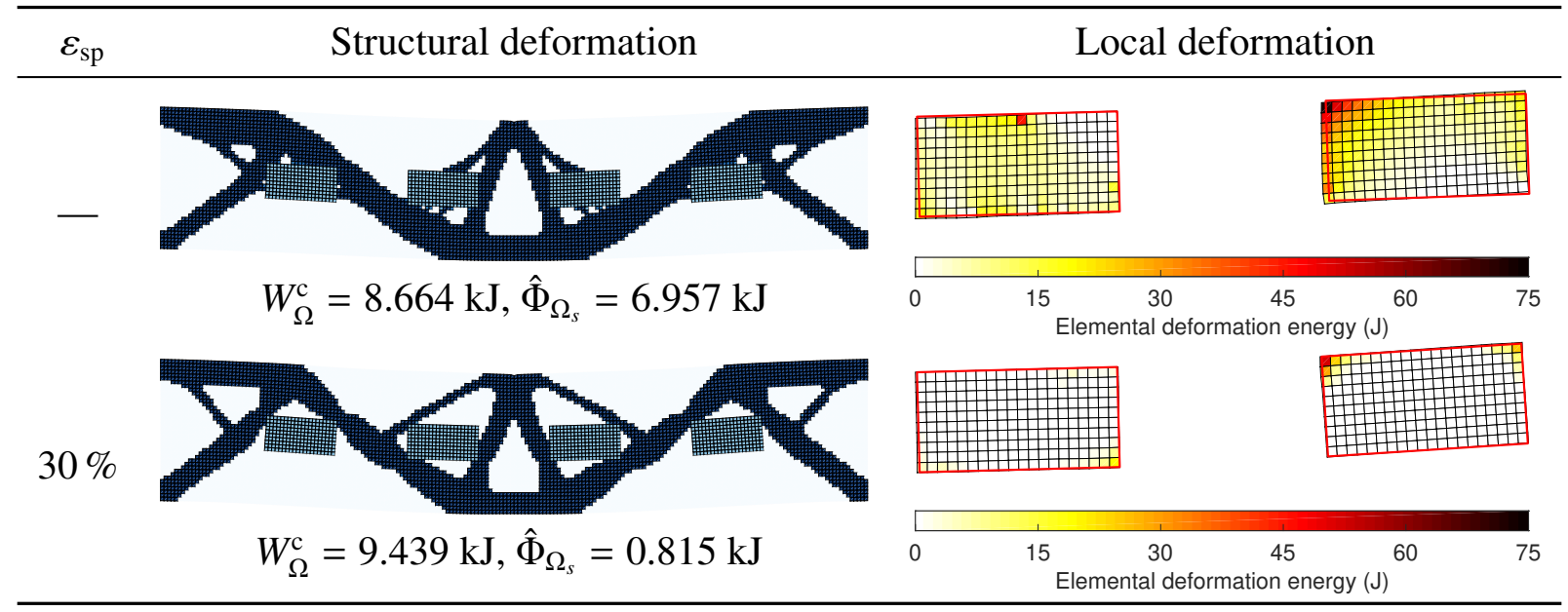

Figure 8: Comparison of thermo-elastic design results with single material and a temperature change of $\Delta T=$ $-100^{\circ} \mathrm{C}$,

While in the shape preserving design results (b), larger external force results in larger displacements but the local shapes are still well preserved. Although some stresses concentrated in the connecting corners, the deformation energies are effectively suppressed in the overall shape preserving domains.

\subsection{Double clamped beam with multiple materials}

In Sec. 5.1, it is presented that the local thermo-elastic warping deformations can be efficiently suppressed by the proposed shape preserving design method using only one material in the design domain. Here, the topology optimization method of multiple materials is further implemented to improve the shape preserving design effect.

The two solid materials used in the multi-material design can be distributed in the design domain with a total volume constraint of $40 \%$. In order to focus on the thermal influence, the mechanical properties of both solids are the same while only the thermal properties are different, i.e. $C T E$ : $\alpha_{1}=20 \times 10^{-6} /{ }^{\circ} \mathrm{C}, \alpha_{2}=10 \times 10^{-6} /{ }^{\circ} \mathrm{C}$. For different temperature changes of $\Delta T=$ $50,100,-100^{\circ} \mathrm{C}$, the multi-material shape preserving design results of the long beam are listed in Figure 12.

As expected, the overall configurations are the same as the results with one material but with better performances. While for different temperature changes, the specific volume fraction of each material (noted as $v f_{1}$ (red), $v f_{2}$ (blue)) are different. Making full use of the material layout under the thermal effect, the designs can manipulate the design material space and the geometrical nonlinearity. Thus, the multi-material topology optimization considering nonlinear thermo-elasticity could further promote capacities for controlling large deformations in the shape preserving design.

\subsection{Planar sandwich panel}

As shown in Figure 13, a planar sandwich panel is considered here. This simplified MBBlike design model is always employed in the elastic supports for thermo-elastic structures [51] and 


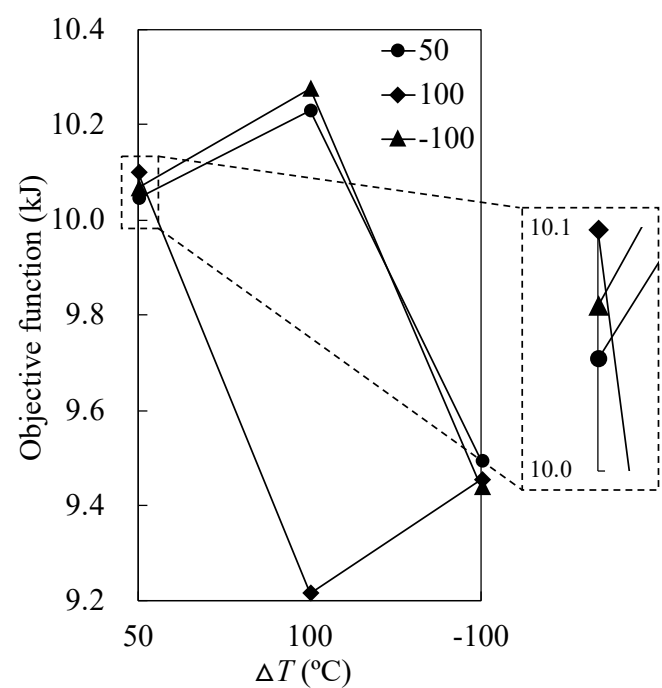

(a) $W_{\Omega}^{\mathrm{c}}$

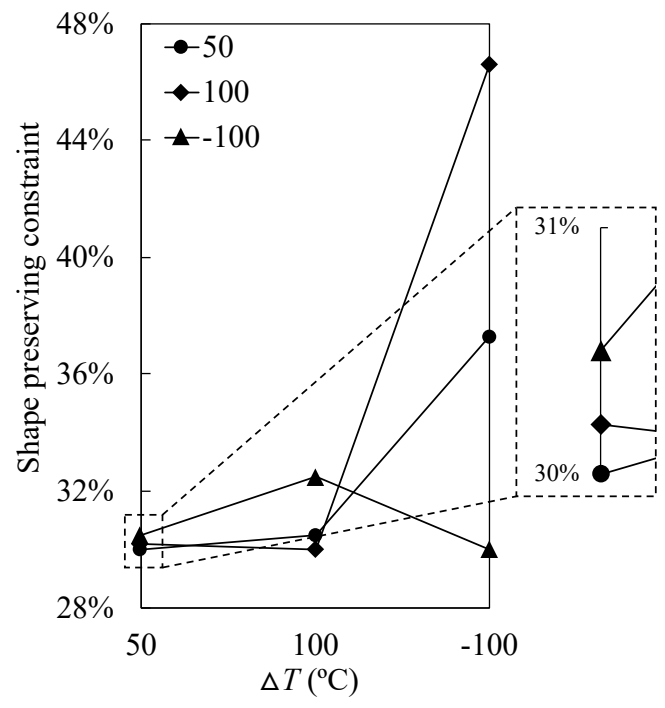

(b) $\varepsilon_{\mathrm{sp}}$

Figure 9: Reanalysis results with different $\Delta T$ for the optimized structures of the shape preserving design

thermal protection system (TPS) [64, 65]. For a structure under thermo-mechanical loads, coupled performances of load bearing and thermal protection are of practical importance. The sandwich panel is covered by two layers of non-designable skins $\Omega_{n d}$ on the top and bottom sides. The skins are functioning sealing and withstanding certain external thermo-mechanical loads, such as hot exhaust gases $[10,13,15,66]$. The core layer is the design domain $\Omega_{d}$, where a reasonabledesigned configuration serves with multiple functions, such as supporting and heat insulation [67].

A rectangular component is embedded in the center of the core layer and set as the shape preserving domain $\Omega_{s}$. The geometric dimensions are marked in Figure 13 and the material properties in different domains are given in Table 1. The height of the skin layer is $1.5 \mathrm{~mm}$ and the thickness of the structure is $5 \mathrm{~mm}$. The panel is subject to a mechanical pressure $p$ evenly distributed at the top boundary (blue line) and a heat flux $q$ at the bottom boundary (red line). The temperature on the top boundary is fixed at $0^{\circ} \mathrm{C}$. The structure is discretized using a mesh of $160 \times 404$-node plane elements.

Table 1: Material properties in different domains of the planar problem model (data source from [68])

\begin{tabular}{ccccc}
\hline Material & $E(\mathrm{GPa})$ & $v$ & $\alpha\left(10^{-6} /{ }^{\circ} \mathrm{C}\right)$ & $K^{\text {th }}\left(\mathrm{W} /\left(\mathrm{m} \cdot{ }^{\circ} \mathrm{C}\right)\right)$ \\
\hline Aluminum $\left(\Omega_{d}\right)$ & 70 & 0.30 & 23.4 & 233 \\
Copper $\left(\Omega_{n d}\right)$ & 120 & 0.34 & 16.5 & 400 \\
Alumina Porcelain $\left(\Omega_{s}\right)$ & 15 & 0.30 & 5.5 & 10 \\
\hline
\end{tabular}

Firstly, the influence of non-uniform temperature field on the structural design is investigated under the mechanical pressure of $p=4 \mathrm{MPa}$. The heat flux is given as $q=4 \mathrm{~kW} / \mathrm{m}^{2}$ and the value of the shape preserving constraint is $\varepsilon_{\mathrm{sp}}=5.0 \%$. To compare the shape preserving effect, both of the standard design and shape preserving design are solved. Corresponding design results 


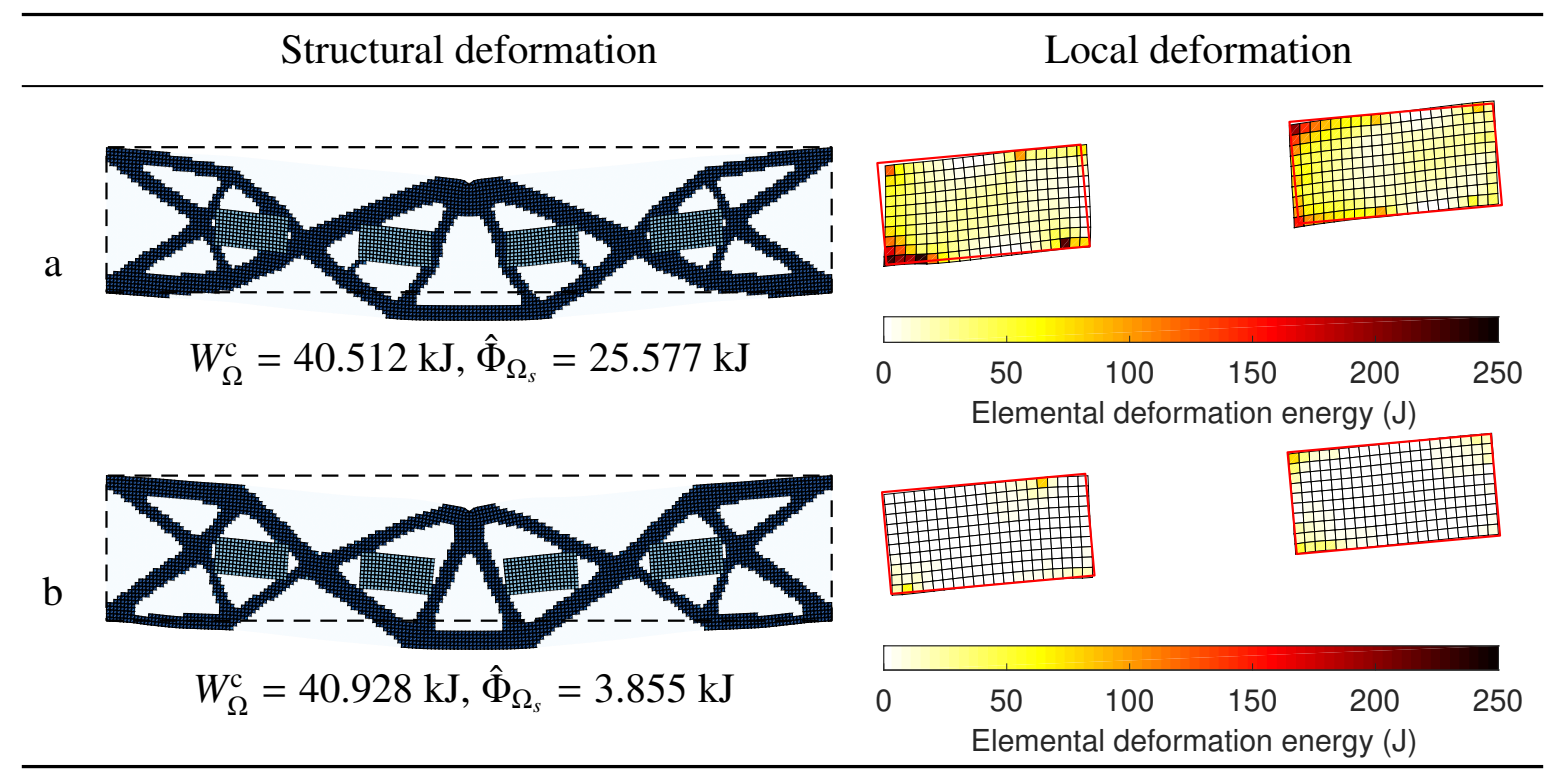

Figure 10: Reanalysis results of optimized structures with a larger force $\left(\Delta T=100^{\circ} \mathrm{C}\right.$ case $)$

and final temperature fields are presented in Figure 14 and the evolution process of the shape preserving design is plotted in Figure 15 with a stable optimization convergence.

For the shape preserving domain in the standard design result, external mechanical loads can be mostly sustained by the main constructions so that the local deformation is not very notable from its geometric shape. However, the elemental deformation energy distribution shows that the standard design result generates large local deformations. It is the significant thermal stress causes this unfavorable behavior. Thus, in the shape preserving design of thermo-elastic structures, improving thermal performances becomes more crucial. The primary factor to be prevented in the shape preserving design, turns into the thermal damage instead of pure mechanical fracture. As shown in the shape preserving design result, more conductive branches are located in the bottom, which efficiently transfers the heat flow away from the local region to the cooler sides. In the thermal field contour plot, the elemental temperature is calculated by the average value of 4 nodal temperatures. Local temperature distribution results are compared and calculated in Figure 16. Nodal temperature difference is measured by the standard deviation $\left(S D_{T}\right)$ with the mean temperature $\left(T_{\text {mean }}\right)$. It is seen that the shape preserving design generates less heat in the shape preserving domain. To further characterize the conductive performance in the thermal field, the global thermal compliance function $C_{\Omega}^{\text {th }}$ and the maximum nodal temperature $T_{\max }$ are commonly used [69]. Here in consistence with the objective function, $C_{\Omega}^{\text {th }}$ is calculated as

$$
C_{\Omega}^{\text {th }}=\frac{1}{2} Q^{\mathrm{T}} \boldsymbol{T} .
$$

Comparing the temperature results of the two designs, the shape preserving design results in a less efficient heat-conductive structure, which means a better heat insulation effect and contributes potentialities in the design of TPS.

Secondly, shape preserving designs are resolved with increased heat flux values of $p=6,8$ 


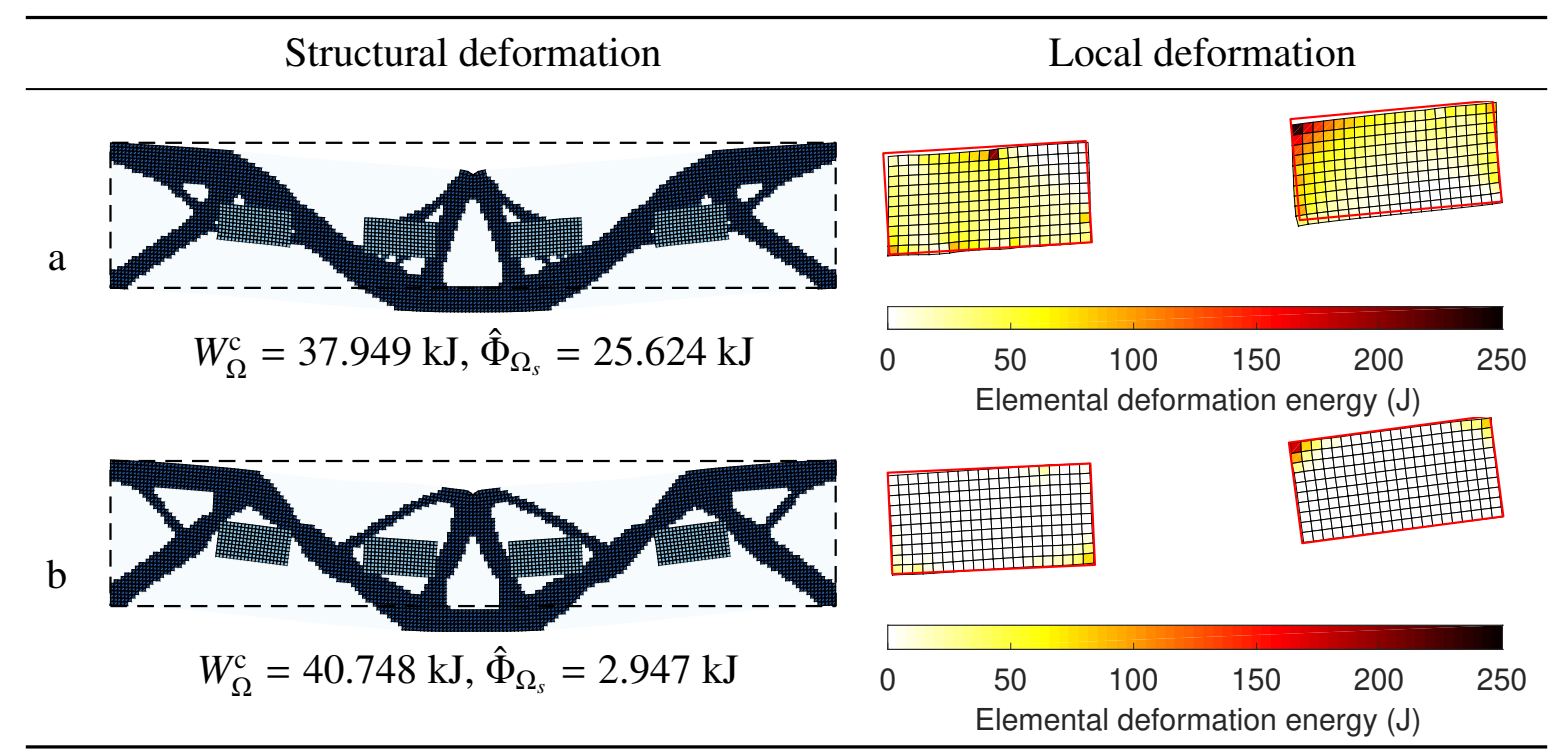

Figure 11: Reanalysis results of optimized structures with a larger force $\left(\Delta T=-100^{\circ} \mathrm{C}\right.$ case $)$

$\mathrm{kW} / \mathrm{m}^{2}$, respectively. The corresponding design results are shown in Figure 17. Evident shape preserving design effects are achieved with low local deformation energies. As the heat is intensified, conductive supporting substructures are also enhanced. A thin straight bar is added underside the shape preserving domain so as to counteracting the bending distortion.

In the case of $q=8 \mathrm{~kW} / \mathrm{m}^{2}$, the maximum nodal temperature in the final temperature result is significantly raised up to $325.5^{\circ} \mathrm{C}$. In this design problem, increased heat flux or thermal effect caused larger deformations. In engineering circumstances, a limited temperature range is often required for normal operation [70]. Thus, the maximum temperature could be further considered in the design process, while the objective function (18b) only concerns the mechanical stiffness. For the possible consideration of thermal conductivity and reducing localized high temperature, a multi-objective design could be applied with

$$
\min (1-w) W_{\Omega}^{\mathrm{c}}+w C_{\Omega}^{\mathrm{th}},
$$

where $w$ is the weight factor between the mechanical and thermal performances. According to the requirement, a thermo-elastic shape preserving design with the multi-objective function will be concurrently optimized for load carrying and temperature cooling.

The same shape preserving design case in Figure 14 is tested here with a multi-objective function and the weight factor is selected as $w=20 \%$. As shown in Figure 18, the maximum nodal temperature falls down in the multi-objective design result. And meanwhile, local deformations are eliminated under the shape preserving constraint. Interestingly, the final objective value of the multi-objective design result is better than the one shown in Figure 14. As the thermal conditions strongly influence the coupled thermal-mechanical performance, the optimization process for such a complex thermo-elastic structure seems to be improved by considering both performances. Two thermo-elastic designs in Figure 14 and 18 may not be directly comparable due to different design conditions, i.e. temperature distributions and local deformations. 


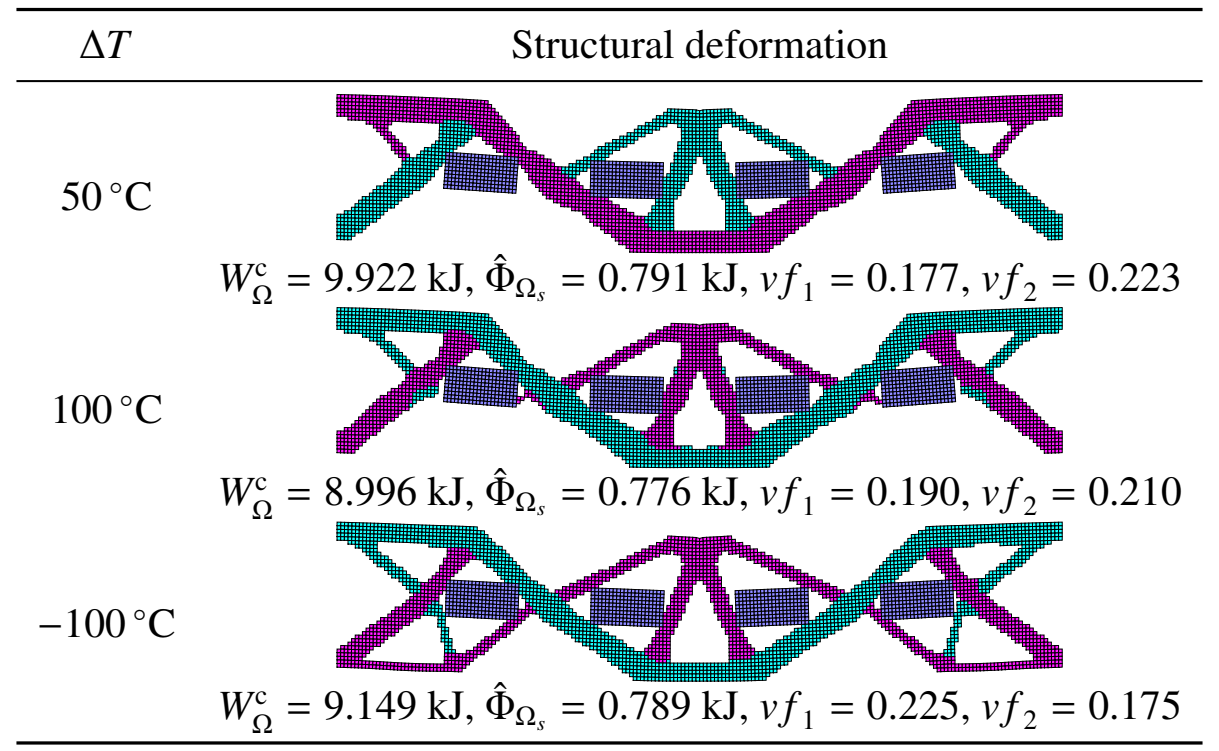

Figure 12: Multi-material shape preserving design results with different temperature changes and a constraint value $\varepsilon_{\mathrm{sp}}=30 \%$

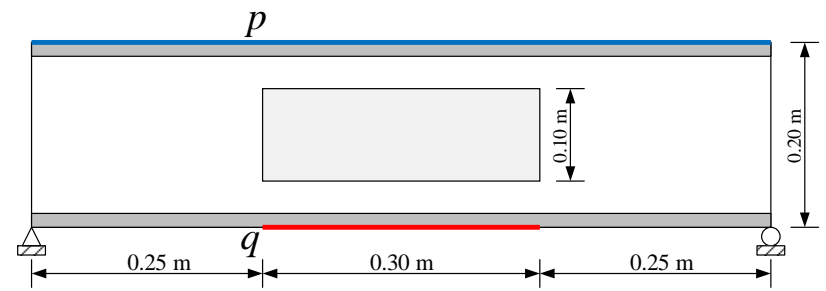

Figure 13: A planar sandwich panel with a rectangular shape preserving domain subject to a mechanical pressure $p$ and a heat flux $q$

At last, to further exhibit the robustness of the proposed shape preserving design method, the geometrical nonlinearity is increased by a mechanical pressure of $p=6 \mathrm{MPa}$. Corresponding design results are shown in Figure 19. Compared with the results in Figure 14, stronger layouts are distributed to support the upper skin undergoing larger loads. The local deformation energy gets larger, but the shape preserving design is still fulfilled with a converged solution in the coupling field. 


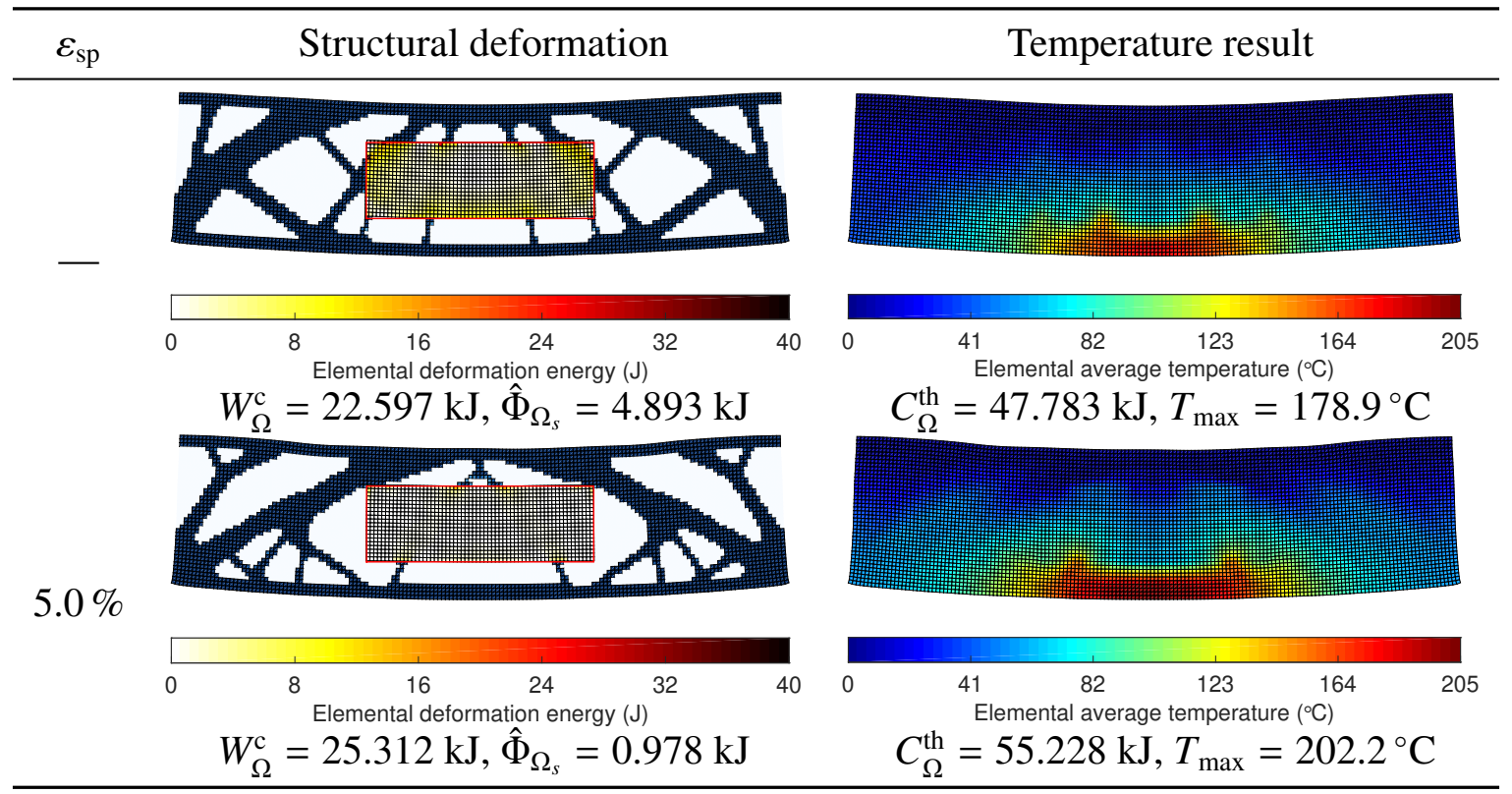

Figure 14: Comparison of thermo-elastic design results for the sandwich panel with a mechanical pressure of $p=4$ $\mathrm{MPa}$ and a heat flux of $q=4 \mathrm{~kW} / \mathrm{m}^{2}$

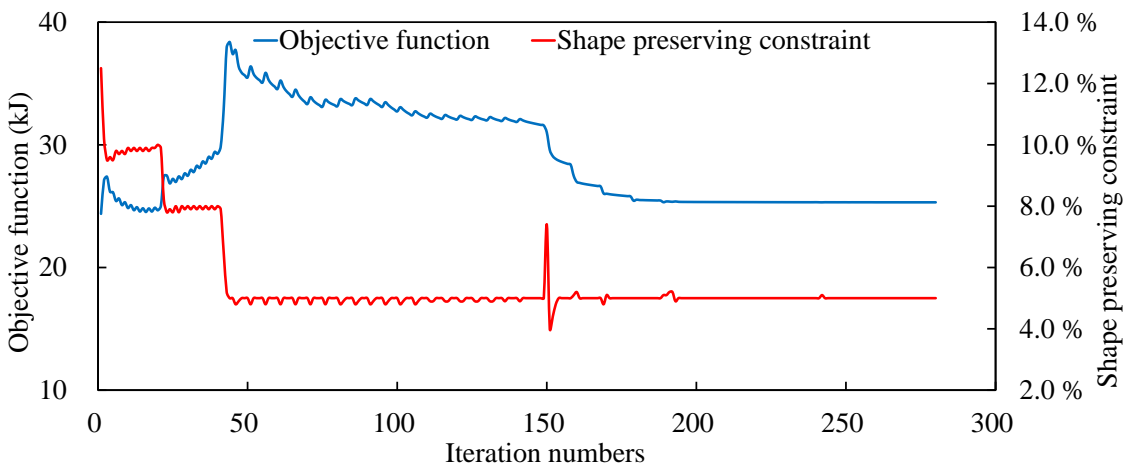

Figure 15: The evolution process of the design functions of shape preserving design result in Figure 14 
Temperature distribution in shape preserving domain

Standard design

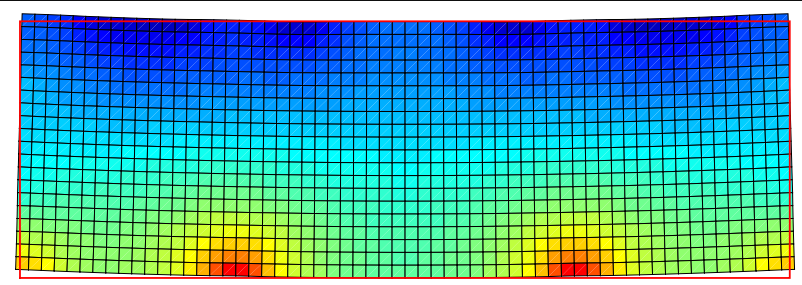

Shape preserving design

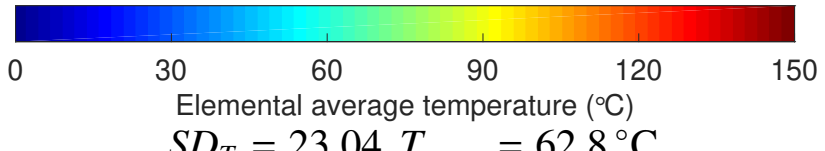

$S D_{T}=23.04, T_{\text {mean }}=62.8^{\circ} \mathrm{C}$
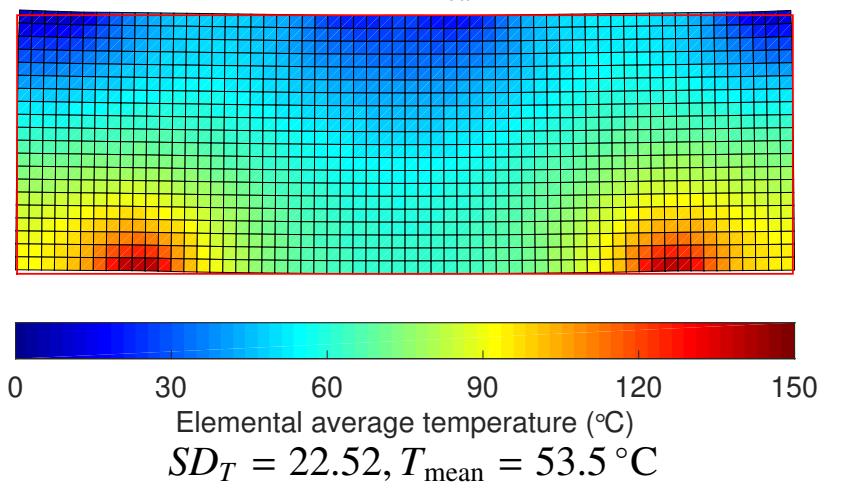

Figure 16: Comparison of the local temperature distribution of thermo-elastic design results

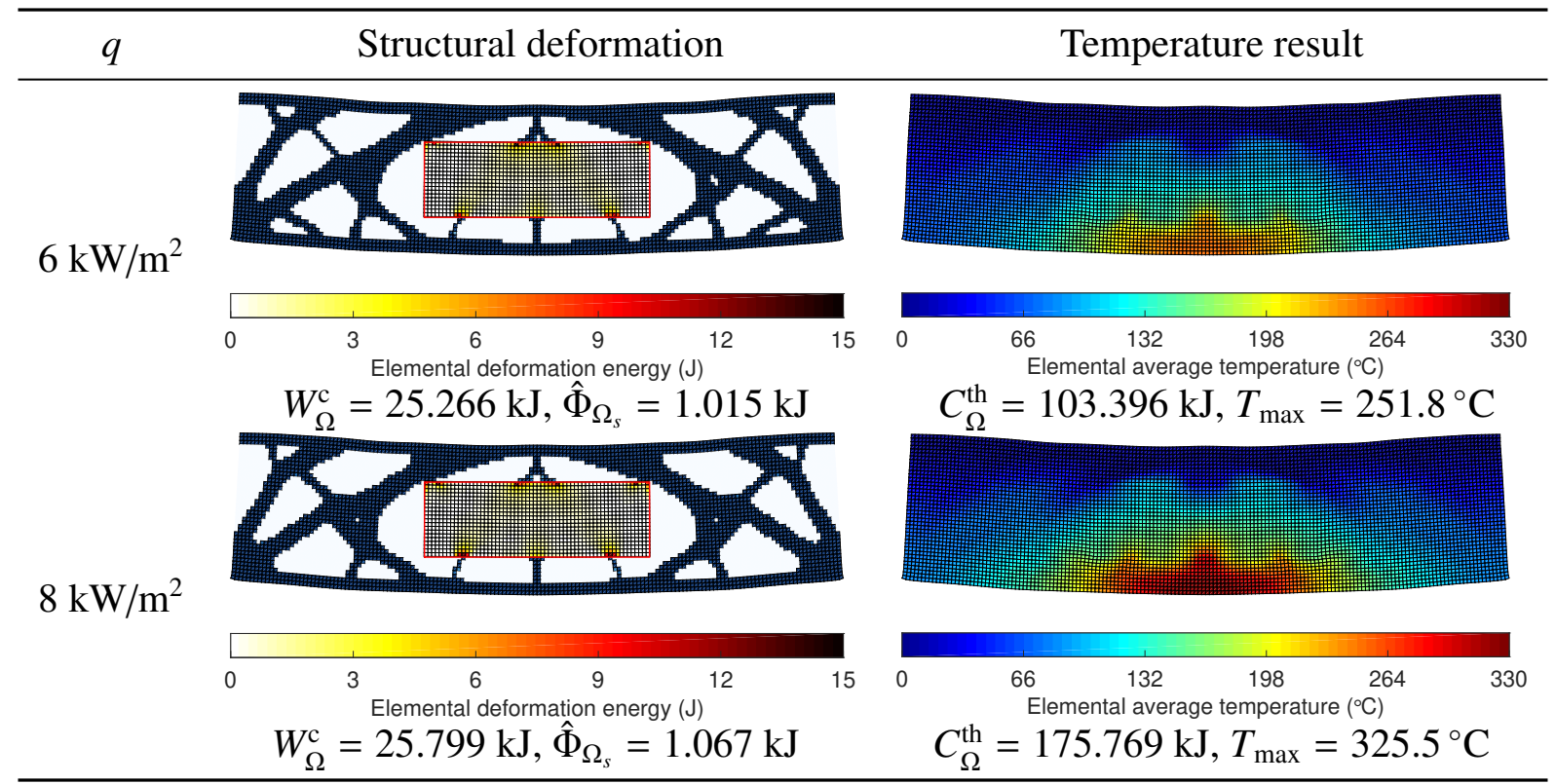

Figure 17: Shape preserving design results for the sandwich panel with different heat flux values under a mechanical pressure of $p=4 \mathrm{MPa}$ and a constraint value $\varepsilon_{\mathrm{sp}}=5.0 \%$ 


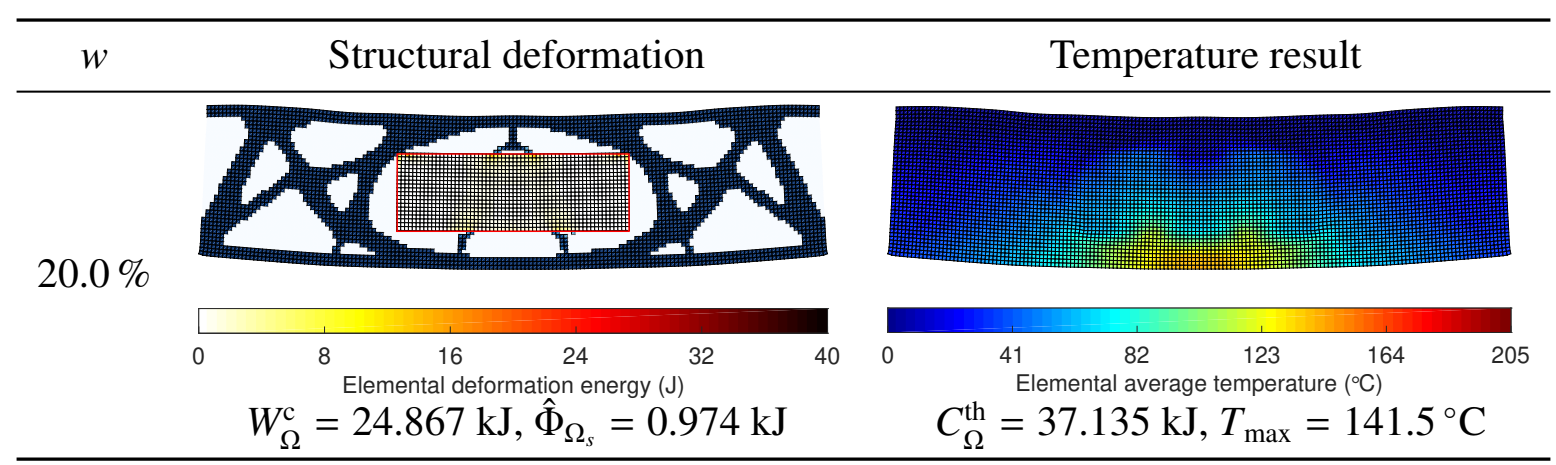

Figure 18: Multi-objective design result for the sandwich panel with a mechanical pressure of $p=4 \mathrm{MPa}$ and a heat flux of $q=4 \mathrm{~kW} / \mathrm{m}^{2}$

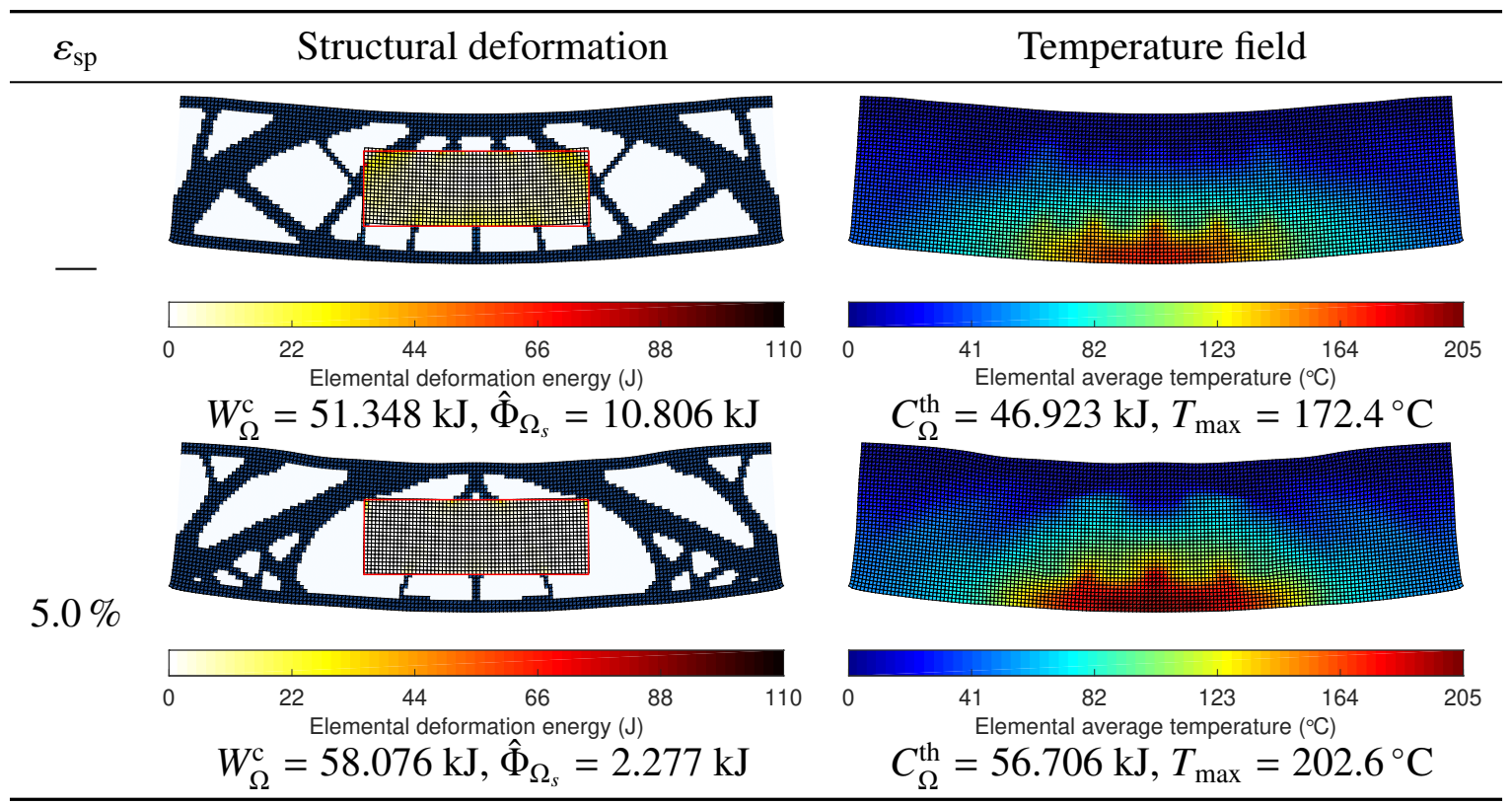

Figure 19: Comparison of thermo-elastic design results for the sandwich panel with a mechanical pressure of $p=6$ $\mathrm{MPa}$ and a heat flux of $q=4 \mathrm{~kW} / \mathrm{m}^{2}$ 


\section{Conclusions}

In this paper, the shape preserving design approach has been extended to the design of thermoelastic structures. Assuming a linear thermal strain, the elastic Green-Lagrange strain is isolated from the total one and derived for large thermo-elastic displacements. Comparing to the existing literature, the nonlinear structural analysis improves the computational accuracy and the design space for practical thermo-elastic problems. Structural complementary elastic work is assigned as the objective function and the integrated deformation energy function is adopted to quantitatively calculate the geometrical distortion over the whole incremental loading process. To avoid thermal damage or warping deformation in shape preserving domains, shape preserving constraint on the local deformation energy is added with a proper relative control value.

In the first example, thermal effects are investigated with a uniform temperature field. It is seen that for the considered loading cases, the thermal deformation is manipulated by the design result, which always counteracts the mechanical loading in both cases of positive and negative temperature changes. And then, the geometrical nonlinearity of the thermo-mechanical coupling structure is also influenced. In the shape preserving design result, local warping deformation is effectively eliminated by the proposed method. To further illustrate the thermal effect and promote the deformation performance, the shape preserving design with multiple materials is carried out with a corresponding modified energy interpolation scheme.

In the second example, a varying temperature field produced by the heat conduction is considered. For such complicated thermo-mechanical conditions, shape preserving design results are well achieved and show some certain thermal insulation effects. A multi-objective design is further tested to reduce hyperthermia in the shape preserving design process.

Above all, the effectiveness of the proposed method is validated. Shape preserving design has demonstrated its capabilities in solving multi-physical and multi-material problems, and possibilities for future extensions and applications.

\section{Replication of results}

For the replication of results, a major program written by MATLAB codes is provided as the supplementary materials of this paper. The codes can be used to reproduce the results of shape preserving designs in Examples section. Several important comments and descriptions are marked as well. Considering the readability and length of the whole codes, some sub-functions, such as the FEA program and MMA subroutines, are not included, which are very common and can be easily implemented by the readers.

\section{Acknowledgement}

The authors would like to thank Ole Sigmund for constructive discussions and also thank anonymous reviewers for their valuable comments. 


\section{Funding Information}

Yu Li received financial support from CSC (China Scholarship Council). Fengwen Wang received support from the Villum foundation through the VILLUM Investigator project InnoTop. This work is also supported by the National Key Research and Development Program (2017YFB1102800) and the National Natural Science Foundation of China (11722219, 11620101002, 51790171, 5171101743).

\section{Compliance with Ethical Standards}

\section{Conflict of interest}

On behalf of all authors, the corresponding author states that there is no conflict of interest.

\section{References}

[1] O. Sigmund, K. Maute, Topology optimization approaches, Structural and Multidisciplinary Optimization 48 (6) (2013) 1031-1055.

[2] J. D. Deaton, R. V. Grandhi, A survey of structural and multidisciplinary continuum topology optimization: post 2000, Structural and Multidisciplinary Optimization 49 (1) (2014) 1-38.

[3] L. Meng, W. Zhang, D. Quan, G. Shi, L. Tang, Y. Hou, P. Breitkopf, J. Zhu, T. Gao, From topology optimization design to additive manufacturing: Today's success and tomorrow's roadmap, Archives of Computational Methods in Engineering (2019) 1-26.

[4] J.-H. Zhu, W.-H. Zhang, L. Xia, Topology optimization in aircraft and aerospace structures design, Archives of Computational Methods in Engineering 23 (4) (2016) 595-622.

[5] N. Aage, E. Andreassen, B. S. Lazarov, O. Sigmund, Giga-voxel computational morphogenesis for structural design, Nature 550 (7674) (2017) 84.

[6] J.-H. Zhu, Y.-B. Zhao, W.-H. Zhang, X.-J. Gu, T. Gao, J. Kong, G.-H. Shi, Y.-J. Xu, D.-L. Quan, Bio-inspired feature-driven topology optimization for rudder structure design, Engineered Science 5 (2019) 46-55.

[7] H. Rodrigues, P. Fernandes, A material based model for topology optimization of thermoelastic structures, International Journal for Numerical Methods in Engineering 38 (12) (1995) 1951-1965.

[8] Q. Xia, M. Y. Wang, Topology optimization of thermoelastic structures using level set method, Computational Mechanics 42 (6) (2008) 837.

[9] T. Gao, W. Zhang, Topology optimization involving thermo-elastic stress loads, Structural and multidisciplinary optimization 42 (5) (2010) 725-738.

[10] J. D. Deaton, R. V. Grandhi, Stiffening of restrained thermal structures via topology optimization, Structural and multidisciplinary optimization 48 (4) (2013) 731-745.

[11] X. Yang, Y. Li, Topology optimization to minimize the dynamic compliance of a bi-material plate in a thermal environment, Structural and Multidisciplinary Optimization 47 (3) (2013) 399-408.

[12] X. Yang, Y. Li, Structural topology optimization on dynamic compliance at resonance frequency in thermal environments, Structural and Multidisciplinary Optimization 49 (1) (2014) 81-91.

[13] J. D. Deaton, R. V. Grandhi, Stress-based design of thermal structures via topology optimization, Structural and Multidisciplinary Optimization 53 (2) (2016) 253-270.

[14] J. Hou, J.-H. Zhu, Q. Li, On the topology optimization of elastic supporting structures under thermomechanical loads, International Journal of Aerospace Engineering 2016.

[15] S. Deng, K. Suresh, Stress constrained thermo-elastic topology optimization with varying temperature fields via augmented topological sensitivity based level-set, Structural and Multidisciplinary Optimization 56 (6) (2017) $1413-1427$.

[16] S. Deng, K. Suresh, Topology optimization under thermo-elastic buckling, Structural and Multidisciplinary Optimization 55 (5) (2017) 1759-1772. 
[17] C. Wu, J. Fang, Q. Li, Multi-material topology optimization for thermal buckling criteria, Computer Methods in Applied Mechanics and Engineering 346 (2019) 1136-1155.

[18] O. Sigmund, Design of multiphysics actuators using topology optimization-part i: One-material structures, Computer methods in applied mechanics and engineering 190 (49-50) (2001) 6577-6604.

[19] O. Sigmund, Design of multiphysics actuators using topology optimization-part ii: Two-material structures, Computer methods in applied mechanics and engineering 190 (49-50) (2001) 6605-6627.

[20] Y.-X. Du, L.-P. Chen, Q.-H. Tian, Z.-J. Wu, Topology synthesis of thermomechanical compliant mechanisms with geometrical nonlinearities using meshless method, Advances in Engineering Software 40 (5) (2009) 315322.

[21] W. M. Rubio, S. Nishiwaki, E. C. N. Silva, Design of compliant mechanisms considering thermal effect compensation and topology optimization, Finite Elements in Analysis and Design 46 (12) (2010) 1049-1060.

[22] J. D. Deaton, R. V. Grandhi, Significance of geometric nonlinearity in the design of thermally loaded structures, in: 56th AIAA/ASCE/AHS/ASC Structures, Structural Dynamics, and Materials Conference, 2015, p. 1431.

[23] Q. Li, G. P. Steven, Y. Xie, Thermoelastic topology optimization for problems with varying temperature fields, Journal of Thermal Stresses 24 (4) (2001) 347-366.

[24] B. Stanford, P. Beran, Aerothermoelastic topology optimization with flutter and buckling metrics, Structural and Multidisciplinary Optimization 48 (1) (2013) 149-171.

[25] A. Diaz, M. Bendsøe, Shape optimization of structures for multiple loading conditions using a homogenization method, Structural optimization 4 (1) (1992) 17-22.

[26] J.-H. Zhu, Y. Li, W.-H. Zhang, J. Hou, Shape preserving design with structural topology optimization, Structural and Multidisciplinary Optimization 53 (4) (2016) 893-906.

[27] Y. Li, J. H. Zhu, W. H. Zhang, L. Wang, Structural topology optimization for directional deformation behavior design with the orthotropic artificial weak element method, Structural and Multidisciplinary Optimization 57 (3) (2018) 1251-1266.

[28] Y. Li, J. Zhu, F. Wang, W. Zhang, O. Sigmund, Shape preserving design of geometrically nonlinear structures using topology optimization, Structural and Multidisciplinary Optimization 59 (4) (2019) 1033-1051.

[29] Dragon / spacex, https://www.spacex.com/dragon.

[30] R. De Borst, M. A. Crisfield, J. J. Remmers, C. V. Verhoosel, Nonlinear finite element analysis of solids and structures, John Wiley \& Sons, 2012.

[31] T. Belytschko, W. K. Liu, B. Moran, K. Elkhodary, Nonlinear finite elements for continua and structures, John wiley \& sons, 2013.

[32] O. C. Zienkiewicz, R. L. Taylor, The finite element method for solid and structural mechanics, Elsevier, 2005.

[33] G. A. Holzapfel, Nonlinear solid mechanics: a continuum approach for engineering science, Meccanica 37 (4) (2002) 489-490.

[34] J. Bonet, R. D. Wood, Nonlinear continuum mechanics for finite element analysis, Cambridge university press, 1997.

[35] T. Bruns, O. Sigmund, Toward the topology design of mechanisms that exhibit snap-through behavior, Computer Methods in Applied Mechanics and Engineering 193 (36-38) (2004) 3973-4000.

[36] R. Stojanovic, S. Djuric, L. Vujosevic, On finite thermal deformations, Arch. Mech. Stosow 16 (1964) $103-108$.

[37] S. Lu, K. Pister, Decomposition of deformation and representation of the free energy function for isotropic thermoelastic solids, International Journal of Solids and Structures 11 (7-8) (1975) 927-934.

[38] L. Vujosevic, V. Lubarda, Finite-strain thermoelasticity based on multiplicative decomposition of deformation gradient, Theoretical and applied mechanics 28 (29) (2002) 379-399.

[39] V. A. Lubarda, Constitutive theories based on the multiplicative decomposition of deformation gradient: Thermoelasticity, elastoplasticity, and biomechanics, Applied Mechanics Reviews 57 (2) (2004) 95-108.

[40] H. Darijani, R. Naghdabadi, Kinematics and kinetics modeling of thermoelastic continua based on the multiplicative decomposition of the deformation gradient, International Journal of Engineering Science 62 (2013) 56-69.

[41] L. Tang, T. Gao, L. Song, L. Meng, C. Zhang, W. Zhang, Topology optimization of nonlinear heat conduction problems involving large temperature gradient, Computer Methods in Applied Mechanics and Engineering 357 (2019) 112600. doi:https://doi.org/10.1016/j.cma.2019.112600. 
URL http://www.sciencedirect.com/science/article/pii/S0045782519304761

[42] P. Michaleris, D. A. Tortorelli, C. A. Vidal, Analysis and optimization of weakly coupled thermoelastoplastic systems with applications to weldment design, International Journal for Numerical Methods in Engineering 38 (8) (1995) 1259-1285.

[43] B. Chen, L. Tong, Thermomechanically coupled sensitivity analysis and design optimization of functionally graded materials, Computer methods in applied mechanics and engineering 194 (18-20) (2005) 1891-1911.

[44] H. Chung, O. Amir, H. A. Kim, Nonlinear thermoelastic topology optimization with the level-set method, in: AIAA Scitech 2019 Forum, 2019, p. 1470.

[45] T. Buhl, C. B. Pedersen, O. Sigmund, Stiffness design of geometrically nonlinear structures using topology optimization, Structural and Multidisciplinary Optimization 19 (2) (2000) 93-104.

[46] G. H. Yoon, Y. Y. Kim, Element connectivity parameterization for topology optimization of geometrically nonlinear structures, International journal of solids and structures 42 (7) (2005) 1983-2009.

[47] F. Wang, B. S. Lazarov, O. Sigmund, J. S. Jensen, Interpolation scheme for fictitious domain techniques and topology optimization of finite strain elastic problems, Computer Methods in Applied Mechanics and Engineering 276 (2014) 453-472.

[48] A. Klarbring, N. Strömberg, Topology optimization of hyperelastic bodies including non-zero prescribed displacements, Structural and Multidisciplinary Optimization 47 (1) (2013) 37-48.

[49] P. Pedersen, N. L. Pedersen, Strength optimized designs of thermoelastic structures, Structural and Multidisciplinary Optimization 42 (5) (2010) 681-691.

[50] P. Pedersen, N. L. Pedersen, Interpolation/penalization applied for strength design of $3 \mathrm{~d}$ thermoelastic structures, Structural and Multidisciplinary Optimization 45 (6) (2012) 773-786.

[51] W. Zhang, J. Yang, Y. Xu, T. Gao, Topology optimization of thermoelastic structures: mean compliance minimization or elastic strain energy minimization, Structural and Multidisciplinary Optimization 49 (3) (2014) 417-429.

[52] E. Andreassen, A. Clausen, M. Schevenels, B. S. Lazarov, O. Sigmund, Efficient topology optimization in matlab using 88 lines of code, Structural and Multidisciplinary Optimization 43 (1) (2011) 1-16.

[53] O. Sigmund, S. Torquato, Design of materials with extreme thermal expansion using a three-phase topology optimization method, Journal of the Mechanics and Physics of Solids 45 (6) (1997) 1037-1067.

[54] X. Wang, Y. Mei, M. Y. Wang, Level-set method for design of multi-phase elastic and thermoelastic materials, International Journal of Mechanics and Materials in Design 1 (3) (2004) 213-239.

[55] O. Sigmund, S. Torquato, Composites with extremal thermal expansion coefficients, Applied Physics Letters 69 (21) (1996) 3203-3205.

[56] O. Sigmund, S. Torquato, Design of smart composite materials using topology optimization, Smart Materials and Structures 8 (3) (1999) 365.

[57] H. Qi, N. Kikuchi, J. Mazumder, Interface study and boundary smoothing on designed composite material microstructures for manufacturing purposes, Structural and multidisciplinary Optimization 26 (5) (2004) 326332.

[58] F. Wang, B. S. Lazarov, O. Sigmund, On projection methods, convergence and robust formulations in topology optimization, Structural and Multidisciplinary Optimization 43 (6) (2011) 767-784.

[59] R. Tavakoli, S. M. Mohseni, Alternating active-phase algorithm for multimaterial topology optimization problems: a 115-line matlab implementation, Structural and Multidisciplinary Optimization 49 (4) (2014) 621-642.

[60] R. Tavakoli, Multimaterial topology optimization by volume constrained allen-cahn system and regularized projected steepest descent method, Computer Methods in Applied Mechanics and Engineering 276 (2014) 534565.

[61] K. Svanberg, The method of moving asymptotes - a new method for structural optimization, International journal for numerical methods in engineering 24 (2) (1987) 359-373.

[62] S. Cho, J.-Y. Choi, Efficient topology optimization of thermo-elasticity problems using coupled field adjoint sensitivity analysis method, Finite Elements in Analysis and Design 41 (15) (2005) 1481-1495.

[63] B. Desmorat, Structural rigidity optimization with an initial design dependent stress field. application to thermoelastic stress loads, European Journal of Mechanics-A/Solids 37 (2013) 150-159.

[64] K. Woo-Young, R. V. Grandhi, M. Haney, An evolutionary optimization method for designing a thermal protec- 
tion system for dynamics, Mechanics based design of structures and machines 34 (1) (2006) 1-24.

[65] T. Pichon, M. Lacoste, R. Barreteau, D. E. Glass, Integrated thermal protection systems and heat resistant structures.

[66] M. A. Haney, Topology optimization of engine exhaust-washed structures, Ph.D. thesis, Wright State University (2006).

[67] E. Gdoutos, A. Shapiro, C. Daraio, Thin and thermally stable periodic metastructures, Experimental mechanics 53 (9) (2013) 1735-1742.

[68] Engineering toolbox, https://www.engineeringtoolbox.com/index.html.

[69] S. Yan, F. Wang, O. Sigmund, On the non-optimality of tree structures for heat conduction, International Journal of Heat and Mass Transfer 122 (2018) 660-680.

[70] E. A. Thornton, Thermal structures for aerospace applications, American Institute of Aeronautics and Astronautics, 1996. 